D. Meredith

Nagoya Math. J.

Vol. 48 (1972), 99-128

\title{
THE FIRST MONSKY-WASHNITZER COHOMOLOGY GROUP
}

\author{
DAVID MEREDITH
}

\section{Introduction}

Throughout this paper, $k$ is a perfect field of characteristic $p>0$, $R$ is a complete discrete valuation ring with residue field $k$ and quotient field of characteristic zero, and $X$ is a connected smooth prescheme of finite type over $k$.

In their papers $[6,8]$ Monsky and Washnitzer have developed a de Rham cohomology theory for certain varieties in characteristic $p$. In particular, they construct functors from the category of "very smooth" $k$ varieties (which includes all affine complete transversal intersections) to the category of $R \otimes_{Z} Q$-vector spaces:

$$
X \sim H^{i}\left(X, R \otimes_{z} Q\right) \quad i \geq 0
$$

which vanishes for $i>\operatorname{dim} X$. We refer to these spaces as the $M W$ cohomology groups.

In this paper we add to the accumulating evidence that Monsky and Washnitzer have defined a "good" cohomology theory. We investigate the functor

$$
X \backsim H^{1}\left(X, R \otimes_{z} Q\right)
$$

and show that this functor may be extended to the category of all smooth $k$-varieties. We prove that:

(1) if $U$ is an open subset of $X$, the assignment

$$
U \backsim H^{1}\left(U, R \otimes_{Z} Q\right)
$$

is a sheaf $\mathscr{H}^{1}$ on $X$;

(2) if $V \subset U$ are open subsets of $X$, then the restriction morphism

Received November 26, 1971. 


$$
H^{1}\left(U, R \otimes_{z} Q\right) \longrightarrow H^{1}\left(V, R \otimes_{Z} Q\right)
$$

is injective;

(3) if $U$ is an open subset of $X$, we show that there is an exact sequence (which is functorial on pairs $(X, U)$ )

$$
\begin{aligned}
0 \longrightarrow & \Gamma\left(X, \mathscr{H}^{1}\right) \stackrel{r}{\longrightarrow} \Gamma\left(U, \mathscr{H}^{1}\right) \longrightarrow G(X, U) \longrightarrow \\
& H^{1}\left(X, \mathscr{H}^{1}\right) \stackrel{r^{\prime}}{\longrightarrow} H^{1}\left(U, \mathscr{H}^{1}\right) \longrightarrow 0
\end{aligned}
$$

where $G(X, U)$ is a finite dimensional $R \otimes_{Z} Q$-vector space and $r$ and $r^{\prime}$ are the restriction morphisms. We show further that $H^{i}\left(X, \mathscr{H}^{1}\right)=0$ for $i \geq 2$.

At the end of this paper we have appended several short applications of the main theory, including a proof that the $M W$-cohomology theory yields "correct" results for algebraic curves: e.g. if $g$ is the genus of a complete curve $X$ then $\operatorname{dim}_{R \otimes_{Z} Q} H^{1}\left(X, R \otimes_{Z} Q\right)=2 g$, and a proof that the group $H^{1}\left(X, R \otimes_{Z} Q\right)$ is a birational invariant of complete varieties $X$.

In a later paper, we will prove that the following theorem: if $X$ is a complete smooth (connected) $k$-variety, and if $\mathscr{D}(X)\left(\operatorname{resp} . \mathscr{D}_{a}(X), \mathscr{D}_{l}(X)\right)$ is the group of divisors on $X$ (resp. the divisors algebraically equivalent to zero, the divisors linearly equivalent to zero) and if

$$
H(X)=\underset{\vec{U}}{\lim } H^{1}\left(U, R \otimes_{\boldsymbol{Z}} Q\right)
$$

as $U$ runs through the open subsets of $X$, then we have natural isomorphisms :

$$
\begin{aligned}
& \frac{H(X)}{H^{1}\left(X, R \otimes_{Z} Q\right)} \cong \mathscr{D}_{a}(X) \otimes_{Z}\left(R \otimes_{Z} Q\right) \\
& H^{1}\left(X, \mathscr{H}^{1}\right) \cong \frac{\mathscr{D}(X)}{\mathscr{D}_{a}(X)} \otimes_{\boldsymbol{Z}}\left(R \otimes_{\boldsymbol{Z}} Q\right)
\end{aligned}
$$

Much of the research upon which this paper is based was completed while I was a graduate student at Brandeis University, where Professor Monsky served as my advisor. I am indebted to him for proposing the initial hypotheses for this work and for providing many valuable suggestions.

\section{Differentials on Weak Formal Preschemes}

In $[5,4.1]$, we defined weak formal $(w f)$ preschemes, which arise in a natural way from $w c f g$ algebras [8]. Suppose $\left(X^{\dagger}, \mathcal{O}_{X}^{\dagger}\right)$ is a $w f$-prescheme. 
Definition 1. The sheaf of differentials, $\Omega_{X^{\dagger} / R}^{\cdot}$, of $\mathcal{O}_{X}^{\dagger}$ is defined on affine $w f$ open sets $U \subset X$ by:

$$
\Gamma\left(U, \Omega_{X^{\dagger} / R}^{\cdot}\right)=D^{\cdot}\left(\Gamma\left(U, \mathcal{O}_{X}^{\dagger}\right)\right),
$$

where $D^{\cdot}\left(\Gamma\left(U, \mathcal{O}_{X}^{\dagger}\right)\right)$ is the module of $\mathfrak{m}$-separated $R$-differentials of $\Gamma\left(U, \mathcal{O}_{X}^{\dagger}\right)$ [8, pp. 196].

The following lemma will prove that $\Omega_{X^{\dagger} / R}$ is a coherent $\mathcal{O}_{X}^{\dagger}$-module.

LemMa 2. Suppose $A^{\dagger}$ is a wefg algebra (over $R$ ) and $f \in A^{\dagger}$. Then $D^{\cdot}\left(A^{\dagger}\right)$ is a finite $A^{\dagger}$-module. Moreover, the natural map $A^{\dagger} \rightarrow A_{[f]}^{\dagger}$ induces an isomorphism:

$$
\psi: D^{\cdot}\left(A^{\dagger}\right) \otimes_{A^{\dagger}} A_{[f]}^{\dagger} \longrightarrow D^{\cdot}\left(A_{[f]}^{\dagger}\right) .
$$

(where $A_{[f]}^{\dagger}=\left(A_{f}^{\dagger}\right)^{\dagger}$.)

Proof. The finiteness of $D^{\cdot}\left(A^{\dagger}\right)$ is proven in [8, Th. 4.5]. $D^{\cdot}\left(A^{\dagger}\right) /\left(\mathrm{m}^{s}\right)$ (resp. $D^{\cdot}\left(A_{[f]}^{\dagger}\right) /\left(\mathfrak{m}^{s}\right)$ ) is the algebra of differentials $D^{\cdot}\left(A^{\dagger} / \mathfrak{m}^{s} A^{\dagger}\right)$ (resp. $\left.D^{\cdot}\left(A_{[f]}^{\dagger} / \mathfrak{m}^{s} A_{[f]}^{\dagger}\right]\right)$ [8, Th. 4.4]. Consequently the $A_{[f]}^{\dagger}$-homomorphism $\psi$ is bijective modulo $\mathrm{m}^{s}$ for all $s>0$. Since $A_{[f]}^{\dagger}$ is a Zariski ring and both $D^{\cdot}\left(A^{\dagger}\right) \otimes_{A} A_{[f]}^{\dagger}$ and $D^{\cdot}\left(A_{[f]}^{\dagger}\right)$ are finite $A_{[f]}^{\dagger}$-modules, $\psi$ is bijective.

The coherent $\mathcal{O}_{X}^{\dagger}$-module $\Omega_{X^{\dagger} / R}^{\cdot}$ is direct sum of components $\Omega_{X^{\dagger} / R}^{i}, i \geq 0$, and there exists the $R$-linear derivation $d: \Omega_{X^{\dagger} / R}^{i} \rightarrow \Omega_{X^{\dagger} / R}^{i+1}$.

DEFinition 3. Let $\left(X^{\dagger}, \mathcal{O}_{X}^{\dagger}\right)$ be a $w f$-prescheme, and let $\Omega_{X^{\dagger} / R}^{\cdot}$ be the sheaf of differentials on $\mathcal{O}_{X}^{\dagger}$. Define a presheaf $\underline{\mathscr{H}}^{i}$ (of $R \otimes_{Z} Q$-modules) on $X^{\dagger}$ by :

$$
\Gamma\left(U, \underline{\mathscr{H}}^{i}\right)=H^{i}\left(\Gamma\left(U, \Omega_{X^{\dagger} / R}^{\cdot} \otimes_{Z} Q\right)\right)
$$

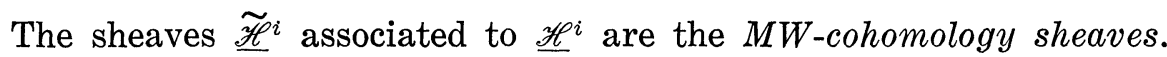

Note that if $X^{\dagger}$ is an affine $w f$-scheme, then since $\Omega_{X^{\dagger} / R}^{i}$ is a coherent $\mathcal{O}_{X}^{\dagger}$-module, $i \geq 0$,

$$
\Gamma\left(X^{\dagger}, \underline{\mathscr{H}}^{i}\right)=\frac{\operatorname{ker} d \cap \Gamma\left(X^{\dagger}, \Omega_{X^{\dagger} / R}^{i}\right)}{\operatorname{im} d \cap \Gamma\left(X^{\dagger}, \Omega_{X^{\dagger} / R}^{i}\right)} \otimes_{Z} Q .
$$

Proposition 4. $\frac{\widetilde{\mathscr{H}}^{0}}{=\underline{\mathscr{H}}^{0}}$; i.e., $\underline{\mathscr{H}}^{0}$ is a sheaf.

Proof. The differentiation map $d: \Omega_{X^{\dagger} / R}^{0} \rightarrow \Omega_{X^{\dagger} / R}^{1}$ is a homomorphism of sheaves, and so $\operatorname{ker} d$ is a sheaf. Since $Q$ is flat over $Z$ and the underlying space is noetherian, $(\operatorname{ker} d) \otimes_{z} Q=\underline{\mathscr{H}}^{0}$ is also a sheaf. 
Proposition 5. Suppose the sheaf $\mathcal{O}_{X}^{+}$is flat over $R$, and that the $k$ prescheme $\left(\bar{X}, \overline{\mathcal{O}}_{X}\right)=\left(X^{\dagger}, \mathcal{O}_{X}^{\dagger} \otimes_{R} k\right)$ is smooth over $k$. Suppose further that $X^{\dagger}$ is irreducible. Let $K$ be the algebraic closure of $R / \mathfrak{m}$ in the function field of $\overline{\mathcal{O}}_{X}$, and let $S$ be a weak formalization of $K$ over $R$. Then $\underline{\mathscr{H}}^{0}$ is canonically isomorphic to the constant sheaf $S \otimes_{Z} Q$.

Proof. Monsky and Washnitzer prove that whenever $U$ is an affine wf open set of $\bar{X}$ such that $\Gamma\left(U, \overline{\mathcal{O}}_{X}\right)$ is very smooth, then $\Gamma\left(U, \underline{\mathscr{H}}^{0}\right)$ is canonically isomorphic to $S \otimes_{Z} Q$ [8, Th. 7.1]. Their proofs also show that this canonical identification commutes with the restriction maps of the sheaf $\underline{\mathscr{H}}^{0}$. Since $\bar{X}$ is smooth, $\bar{X}$ has a neighborhood basis of very smooth open sets. Consequently $\underline{\mathscr{H}}^{0}$ is canonically isomorphic to the constant sheaf with stalk $S \otimes_{Z} Q$.

This paper devoted primarily to a sheaf closely related to $\underline{\mathscr{H}}^{1}$. The following proposition will be essential.

Proposition 6. Suppose $\mathcal{O}_{X}^{+}$is flat over $R$ and the k-prescheme $\left(X^{\dagger}, \mathcal{O}_{X}^{\dagger} \otimes_{R} k\right)$ is smooth over $k$. Then $\underline{\mathscr{H}}^{1}=\underline{\mathscr{H}}^{1}$; i.e., $\underline{\mathscr{H}}^{1}$ is a sheaf.

Proof. Let $B_{1}$ (resp. $Z_{1}$ ) be the presheaf of exact (resp. closed) oneforms in $\Omega_{X^{\dagger} / R}^{1} . \quad B_{1}$ and $Z_{1}$ are defined on affine $w f$ open sets of $X$. We have two exact sequences of presheaves:

$$
\begin{aligned}
& 0 \longrightarrow \mathscr{H}^{0} \longrightarrow \mathcal{O}_{X}^{\dagger} \otimes_{Z} Q \longrightarrow B_{1} \otimes_{Z} Q \longrightarrow 0 \\
& 0 \longrightarrow B_{1} \otimes_{Z} Q \longrightarrow Z_{1} \otimes_{Z} Q \longrightarrow \mathscr{H}^{1} \longrightarrow 0
\end{aligned}
$$

$\mathcal{O}_{X}^{\dagger} \otimes_{Z} Q$ is a sheaf and $\underline{\mathscr{H}}^{0}$ is a sheaf with $H^{i}\left(U, \mathscr{H}^{0}\right)=0$ for any open set $U$ and $i>0$. Consequently $B_{1} \otimes_{z} Q$ is a sheaf. Further, for affine wf open sets $U \subset X^{\dagger}, H^{1}\left(U, B_{1} \otimes_{Z} Q\right)=0$ because $H^{1}\left(U, \mathcal{O}_{X}^{\dagger} \otimes_{Z} Q\right)=0$. Also, $Z_{1} \otimes_{Z} Q$ is a sheaf, because it is the kernel of the sheaf map $d \otimes 1: \Omega_{X^{\dagger} / R}^{1} \otimes_{Z} Q \rightarrow \Omega_{X^{\dagger} / R}^{2} \otimes_{Z} Q$.

Thus, for $U$ an affine $w f$ open set of $X, \Gamma\left(U, \underline{\mathscr{H}}^{1}\right)=\Gamma\left(U, \underline{\mathscr{H}}^{1}\right)$, which proves that $\underline{\mathscr{H}}^{1}$ is a sheaf.

\section{Definitions and Elementary Properties}

Recall that $X$ is a smooth scheme of finite type over $k$. If $U \subset X$ is an affine open set and $\Gamma\left(U, \mathcal{O}_{X}\right)$ is very smooth, we shall say that $U$ is very smooth affine. For the definition of very smooth, see [8, Def. 3.1]. A complete transversal intersection [8, Def, 3.3] is very smooth affine 
[8, Th. 3.6], and a very smooth affine open set can be covered by principal open subsets which are complete transversal intersections. Also, very smooth affine open sets are absolutely non-singular [8, Th. 2.5 et. passim., also $\left.2,0_{\text {IV }}, 22.5 .8\right]$.

If $U \subset X$ is a very smooth affine open set, we may associate to $U$ the Monsky-Washnitzer cohomology groups $H^{i}\left(\Gamma\left(U, \mathcal{O}_{X}\right), R \otimes_{Z} Q\right)$ [8, Th. 5.6]. If $V \subset U$ are both very smooth affines, then the restriction morphism $\Gamma\left(U, \mathcal{O}_{X}\right) \rightarrow \Gamma\left(V, \mathcal{O}_{X}\right)$ induces a unique homomorphism $H^{i}\left(\Gamma\left(U, \mathcal{O}_{X}\right), R \otimes_{Z} Q\right)$ $\rightarrow H^{i}\left(\Gamma\left(V, \mathcal{O}_{X}\right), R \otimes_{Z} Q\right)$.

DEFINITION $1 . \mathscr{H}^{i}$ is the presheaf of $R \otimes_{Z} Q$-modules defined on very smooth affine open sets $U \subset X$ by:

$$
\Gamma\left(U, \mathscr{H}^{i}\right)=H^{i}\left(\Gamma\left(U, \mathcal{O}_{X}\right), R \otimes_{Z} Q\right) .
$$

$\mathscr{H}^{i}$ is the $i$-th $M-W$ (Monsky-Washnitzer) presheaf.

Since any principal open subset of a very smooth affine open set is a very smooth affine $\mathscr{H}^{i}$ is defined on a basis of the topology of $X$.

If $\left(X, \mathcal{O}_{X}\right)$ is the reduction modulo $n$ of a $w f$ prescheme $\left(X^{\dagger}, \mathcal{O}_{X}^{\dagger}\right)$ flat over $R$ [5, 4.1], and if $\Omega_{X^{\dagger} / R}^{\cdot}$ is the sheaf on continuous $R$-differentials of $\mathcal{O}_{X}^{\dagger}$, then $X$ may be identified with $X^{\dagger}$, and the presheaf $\mathscr{H}^{i}$ (1.3) is canonically isomorphic to $\mathscr{H}^{i}$ on those open subsets where $\mathscr{H}^{i}$ is defined. Thus the sheaves $\underline{\mathscr{H}}^{i}$ and $\widetilde{\mathscr{H}}^{i}$ are canonically isomorphic all $i \geq 0$.

Although $\left(X, \mathcal{O}_{X}\right)$ may not be liftable to a smooth $w f$ prescheme over $R$, the existence of liftings for very smooth open subschemes of $X$ leads to a proof of the following theorem.

Proposition 2. $\mathscr{H}^{0}$ and $\mathscr{H}^{1}$ are both sheaves.

Let $K$ be the algebraic closure of $k$ in $\Gamma\left(X, \mathcal{O}_{X}\right) . \quad K$ is separable and finite over $k$. Consequently $K$ has a weak formalization $S$ which is a discrete valuation ring and a finite extension of $R$ [8, Lemma 7.2].

Proposition 3. $\mathscr{H}^{0}$ is a constant sheaf isomorphic to $S \otimes_{Z} Q$.

Proof. Since any two open sets of $X$ have non-empty intersection, we may assume that $X$ itself is very smooth affine. In this case the theorem follows trivially from (1.5).

\section{The Residue Map}

Suppose $A$ is a very smooth $k$-algebra and $t \in A$ is not a unit or a zero-divisor. Monsky has defined the notion of an admissible pair [6, Def. 
4.1]; an example of such a pair is $(A, t)$ if $A$ and $A /(t)$ are both complete transversal intersections.

DeFinition 1. Let $U \subset X$ be an open affine set, and let $D \subset X$ be a hypersurface (i.e. a reduced subprescheme of pure codimension 1 in $X$ ). $U$ is an admissible neighborhood of $D$ if and only if :

(1) the ideal $I(D \cap U)$ is principal on some $t \in \Gamma\left(U, \mathcal{O}_{X}\right)$;

(2) $\left(\Gamma\left(U, \mathcal{O}_{X}\right), t\right)$ is an admissible pair.

If $s, t \in \Gamma\left(U, \mathcal{O}_{X}\right)$ each generate $I(D \cap U)$, and if $\left(\Gamma\left(U, \mathcal{O}_{X}\right), t\right)$ is an admissible pair, then $\left(\Gamma\left(U, \mathcal{O}_{X}\right), s\right)$ is an admissible pair. That is, $U$ being an admissible neighborhood of $D$ is independent of the choice of generator for $I(D \cap U)$.

Suppose $U$ is an admissible neighborhood of $D$, and $V$ is a principal open subset of $U$ such that $V \cap D \neq \emptyset$. Then $V$ is an admissible neighborhood of $D$ [8, Th. 3.5]. Thus, if a point $p \in D$ is contained in an admissible neighborhood of $D$, there is a neighborhood basis at $p$ consisting of admissible neighborhoods of $D$.

Notice that if $U$ is an admissible neighborhood of $D$, then $U$ is an admissible neighborhood of any (reducible or irreducible) component of $D$ which has non-empty intersection with $U$ and which is principal on $U$. For if $E$ is a component of $D$, then since $\Gamma\left(U \cap D, \mathcal{O}_{D}\right)$ is very smooth, $\Gamma\left(U \cap E, \mathcal{O}_{E}\right)$ is a direct summand of $\Gamma\left(U \cap D, \mathcal{O}_{D}\right)$ and consequently is very smooth [8, Cor. to Th. 3.5].

Proposition 2. Let $U \subset X$ be an affine complete transversal intersection, and let $D$ be a hypersurface on $X$. Suppose $p_{1}, \cdots, p_{r} \in D \cap U$ are non-singular on $D$. Then there exists an admissible neighborhood $V$ of $D$ such that $V \subset U$ and $p_{i} \in V, i=1, \cdots, r$.

Proof. There is, in fact, a principal open subset $V$ of $U$ such that:

(1) $p_{i} \in V$;

(2) $D \cap V$ is non-singular;

(3) $I(D \cap V)$ is principal in $\Gamma\left(V, \mathcal{O}_{X}\right)$.

To find $V$, let $\mathfrak{p}_{i}=I\left(p_{i}\right) \subset \Gamma\left(U, \mathcal{O}_{X}\right)$, and let $a$ be the ideal of the singular subvariety of $D \cap U$ pulled back to $\Gamma\left(U, \mathcal{O}_{X}\right)$. Let $S=\Gamma\left(U, \mathcal{O}_{X}\right) \sim \bigcup_{i=1}^{r} \mathfrak{p}_{i}$. Since $\mathfrak{a} \not \subset \mathfrak{p}_{i}, \mathfrak{a} \not \subset \cup_{i=1}^{r} \mathfrak{p}_{i}$. Thus there is an element $f \in \mathfrak{a} \cap S$. Also, $\Gamma\left(U, \mathcal{O}_{X}\right)_{S}$ is a regular semi-local ring and so is a PID. Consequently 
there is an element $g \in S$ such that $I(D \cap U)_{g}$ is principal. Let $V=U_{f g}$. $V$ satisfies conditions (1), (2), and (3) above.

$\Gamma\left(V, \mathcal{O}_{X}\right)$ is a complete transversal intersection, $\Gamma\left(V \cap D, \mathcal{O}_{D}\right)=$ $\Gamma\left(V, \mathcal{O}_{X}\right) / I(D \cap V)$ is regular, and $I(D \cap V)$ is principal. Since $k$ is perfect, these three conditions imply that $\Gamma\left(V \cap D, \mathcal{O}_{D}\right)$ is a complete transversal intersection. Thus $V$ is an admissible neighborhood of $D$ satisfying the proposition.

CoRollary 3. Suppose $D$ is a hypersurface on $X$ and $p$ is a nonsingular point of $D$. There is an $X$-neighborhood of $p$ which is an admissible neighborhood of $D$.

Proof. Choose $U \subset X$ to be an affine complete transversal intersection which contains $p$. Proposition 2 gives a neighborhood $V \subset U$ of $p$ satisfying the corollary.

Corollary 4. Let $U \subset X$ be open and suppose $D$ is a hypersurface on $X$. Then there is an admissible neighborhood $V$ of $D$ such that $V \sim D \subset U$. Further, if $U \cap D \neq \emptyset$, we can choose $V \subset U$.

Proof. Suppose $U \cap D \neq \emptyset$. Choose a point $p \in U \cap D$ which is regular on $D$, and let $W$ be an admissible neighborhood of $D$ which contains $p$. Take $V$ to be a principal open subset of $W$ such that $p \in V \subset U$. $V$ is an admissible neighborhood of $D$.

In general, select an admissible neighborhood $W$ of $D$. Such admissible neighborhoods exist by Corollary 3. Let $A=\Gamma\left(W, \mathcal{O}_{X}\right)$ and $I(D \cap W)$ $=t A$. Choose $s \in A \sim t A$ such that $W_{s t} \subset U$. This is possible because $W \cap U \neq \emptyset$, as $X$ is irreducible. Let $V=W_{s} . \quad V$ is an admissible neighborhood of $D$, and $V \sim D=V_{t} \subset U$.

For an admissible pair $(A, t)$, Monsky has defined the residue map:

$$
\text { res : } H^{1}\left(A_{t}, R \otimes_{z} Q\right) \longrightarrow H^{0}\left(\frac{A}{t A}, R \otimes_{z} Q\right) \text {. }
$$

This homomorphism is part of the long, exact Gysin sequence:

$$
\cdots \longrightarrow H^{i}(A, K) \longrightarrow H^{i}\left(A_{t}, K\right) \longrightarrow H^{i-1}\left(\frac{A}{(t)}, K\right) \longrightarrow H^{i+1}(A, K) \longrightarrow \cdots
$$

where $K=R \otimes_{z} Q$.

Using the Gysin sequence, we will define the residue of a local section of the sheaf $\mathscr{H}^{1}$ with respect to a prime divisor (i.e. an irreducible hyper- 
surface) on $X$. The idea is to map sections of $\mathscr{H}^{1}$ to the "zero'th $M-W$ cohomology" of $D$ in such a way that the kernel of this map consists precisely of those sections which extend to points of $D$. First we must specify what we mean by the "zero'th $M-W$ cohomology" of $D$, as $D$ is not in general a smooth variety.

Fix a prime divisor $D$ on $X$, and let $E \subset D$ be the maximal smooth open subvariety of $D . E$ is an irreducible $k$-prescheme of finite type. Let $K_{D}$ be the algebraic closure of $k$ in $\Gamma\left(E, \mathcal{O}_{E}\right)$. Since $k$ is perfect, we may set $S_{D}=$ the weak formalization of $K_{D}$ over $R$. The next lemma shows that $S_{D} \otimes_{Z} Q$ functions as the "zero'th $M-W$ cohomology group" of $D$.

Lemma 5. Suppose $U \subset D$ is a very smooth affine set. Then $S_{D} \otimes_{Z} Q$ $=H^{0}\left(\Gamma\left(U, \mathcal{O}_{D}\right), R \otimes_{Z} Q\right)$.

Proof. Since $U$ is smooth, $U \subset E . E$ is connected, and so $K_{D}$ is the algebraic closure of $k$ in $\Gamma\left(U, \mathcal{O}_{D}\right)$. The lemma is then a consequence of (2.3).

Definition 6. Let $i: D \rightarrow X$ be the inclusion map. Let $\tilde{K}_{D}$ denote the constant sheaf $S_{D} \otimes_{Z} Q$ on $X$. Define $\mathscr{H}_{D}^{0}=i_{*} \circ i^{*}\left(\tilde{K}_{D}\right)$, and define $\mathscr{S}_{D}$ to be the kernel of the canonical surjection $\tilde{K}_{D} \rightarrow \mathscr{H}_{D}^{0}$.

$\mathscr{H}_{D}^{0}$ is the extension by zero of the constant sheaf $S_{D} \otimes_{Z} Q$ on $D$, and we have an exact sequence:

$$
0 \longrightarrow \mathscr{S}_{D} \longrightarrow \tilde{K}_{D} \longrightarrow \mathscr{H}_{D}^{0} \longrightarrow 0 \text {. }
$$

Proposition 7. If $U \subset X$ is open, $\Gamma\left(U, \mathscr{S}_{D}\right)=0$ for almost all prime divisors $D$.

Proof. $X$ is a noetherian space, so $X \sim U$ contains at most a finite number of prime divisors $D . \quad \Gamma\left(U, \mathscr{S}_{D}\right) \neq 0$ if and only if $D \cap U=\emptyset$, so $\Gamma\left(U, \mathscr{S}_{D}\right)=0$ for almost all prime divisors $D$.

Suppose that $V$ is an admissible neighborhood of a prime divisor $D$. By Lemma 5 and the Gysin sequence, Monsky's residue map is a homomorphism :

$$
\operatorname{res}_{D}: \Gamma\left(V \sim D, \mathscr{H}^{1}\right) \longrightarrow \Gamma\left(V \sim D, \mathscr{S}_{D}\right) .
$$

If $\psi$ is the restriction map, the following sequence is the initial portion of the Gysin sequence, and thus is exact: 


$$
0 \longrightarrow \Gamma\left(V, \mathscr{H}^{1}\right) \stackrel{\psi}{\longrightarrow} \Gamma\left(V \sim D, \mathscr{H}^{1}\right) \stackrel{\operatorname{res}_{D}}{\longrightarrow} \Gamma\left(V \sim D, \mathscr{S}_{D}\right) .
$$

The remainder of this section is devoted to extending the homomorphism $\operatorname{res}_{D}$ to a morphism of sheaves,

$$
\operatorname{res}_{D}: \mathscr{H}^{1} \longrightarrow \mathscr{S}_{D} \text {. }
$$

For notational purposes, $D$ will continue to denote some fixed prime divisor on $X$.

LEMma 8. Suppose $W \subset V$ are admissible neighborhoods of $D$. Then the following diagram commutes (where the vertical arrows are restriction maps):

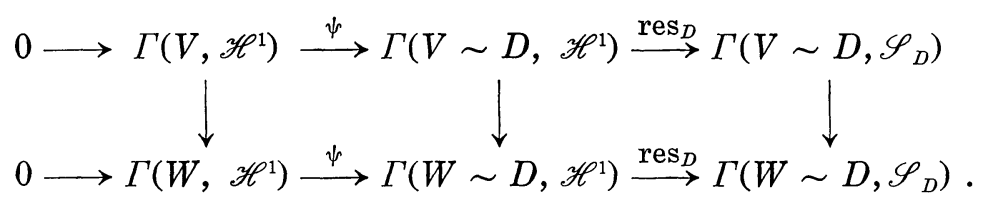

Proof. Let $A=\Gamma\left(V, \mathcal{O}_{X}\right), B=\Gamma\left(W, \mathcal{O}_{X}\right), s A=I(V \cap D)$, and $t A=$ $I(W \cap D)$. The restriction homomorphism $A \rightarrow B$ induces a map of admissible pairs $(A, s) \rightarrow(B, t)$ which has ramification degree one [6, Def. 4.2]; consequently the lemma follows from Lemma 5 and [6, Th. 4.3].

DeFinition 9. Define the morphism

$$
\operatorname{res}_{D}: \mathscr{H}^{1} \longrightarrow \mathscr{S}_{D}
$$

as follows: If $U \subset X$ is open, choose $V$ so that $V$ is an admissible neighborhood of $D$ and $V \sim D \subset U$ (Corollary 4). The homomorphism

$$
\operatorname{res}_{D}: \Gamma\left(V \sim D, \mathscr{H}^{1}\right) \longrightarrow \Gamma\left(V \sim D, \mathscr{S}_{D}\right)
$$

is Monsky's residue map. Extend $\operatorname{res}_{D}$ to $U$ in such a way that the following square commutes (where the vertical arrows denote restriction maps):

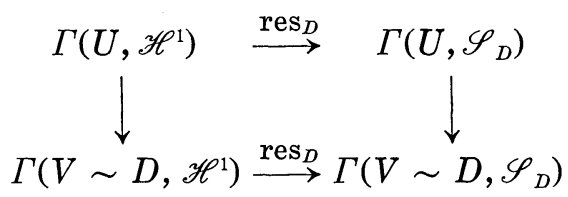

\section{A Theorem on Residues}

Retain all of the notation of $\S 3$. 
The main business of this section is to prove the following theorem: if $D$ is a prime divisor on $X$ and $p \in D$, then there is an $X$-neighborhood $U$ of $p$ such that $\operatorname{res}_{D}: \Gamma\left(U \sim D, \mathscr{H}^{1}\right) \rightarrow \Gamma\left(U \sim D, \mathscr{S}_{D}\right)$ is surjective. Thus we can find a cohomology class on $U \sim D$ which has a prescribed residue at $D$ and zero residue on every other prime divisor passing through $p$. In fact, our proof will show that every very smooth affine neighborhood $U$ of $p$ such that each "absolutely irreducible" component of $D$ is globally principal on $U$ has the desired property.

We will first prove the theorem under the additional assumption that $D$ is "absolutely irreducible". i.e. $K_{D}=k$. Then we will construct an appropriate neighborhood $U$ of $p$ and a field extension $K$ of $k$ such that the components of $K \times_{k} D$ are absolutely irreducible. Given a residue on $D$, we will construct a cohomology class on $K \times{ }_{k} U \sim K \times{ }_{k} D$ which, when pushed down to $U \sim D$, has the required residue.

Proposition 1. Let $(A, t)$ be an admissible pair. There exists $\sigma \in H^{1}\left(A, R \otimes_{Z} Q\right)$ such that $\operatorname{res}(\sigma)=1$.

Proof. Let $A^{\dagger}$ be a weak formalization of $A$, let $B^{\dagger}$ be a weak formalization of $A_{t}$, and let $T$ be a preimage of $t$ in $A^{\dagger}$. The differential $d T / T \in D^{1}\left(B^{\dagger}\right)$ is clearly closed. Monsky's map $\lambda_{t}: D^{0}\left(A^{\dagger} / T A^{\dagger}\right) \rightarrow$ $D^{1}\left(B^{\dagger}\right) / D^{1}\left(A^{\dagger}\right)$, which is a homology isomorphism, yields $\lambda_{t}(1)=d T / T \bmod$ - $D^{1}\left(A^{\dagger}\right)$. Thus res $(d T / T)=1$. [cf. 6, Th. 1.5, Th. 4.1]

CoRollary 2. Let $D$ be a prime divisor on $X$ and $p \in D$. Suppose further that $K_{D}=k$. Then for every very smooth affine neighborhood $U$ of $p$ such that $D \cap U$ is principal on $U$, the residue homomorphism:

$$
\operatorname{res}_{D}: \Gamma\left(U \sim D, \mathscr{H}^{1}\right) \longrightarrow \Gamma\left(U \sim D, \mathscr{S}_{D}\right)
$$

is surjective.

Proof. Let $t \in \Gamma\left(U, \mathcal{O}_{X}\right)$ generate $I(D \cap U)$. Select an admissible neighborhood $V$ of $D$ such that $V \subset U$ (3.4). If $T$ is the preimage of $t$ in some weak formalization of $\Gamma\left(U, \mathcal{O}_{X}\right)$, then the differential $d T / T$ represents a cohomology class $\sigma \in \Gamma\left(U \sim D, \mathscr{H}^{1}\right)$. The proof of Proposition 1 shows that if $\tau$ is the restriction of $\sigma$ to $\Gamma\left(V \sim D, \mathscr{H}^{1}\right)$, then $\operatorname{res}_{D} \tau=1 \in \Gamma\left(V \sim D, \mathscr{S}_{D}\right)$. Consequently $\operatorname{res}_{D} \sigma=1 \in \Gamma\left(U \sim D, \mathscr{S}_{D}\right)$. Since $\Gamma\left(U \sim D, \mathscr{S}_{D}\right)=R \otimes_{Z} Q$ by assumption, $\operatorname{res}_{D}$ is surjective.

Having proven a special case of our theorem, we need now to study 
the effect of ground field extension on the cohomology sheaves. In order to study extension of the ground field, we must give a further sheaftheoretic interpretation of Monsky's residue map. In particular, we must clarify the notion of a residue at a not necessarily irreducible hypersurface.

If $U$ is an admissible neighborhood of a prime divisor $D$, let $A=\Gamma\left(U, \mathcal{O}_{X}\right)$ and $t A=I(D \cap U)$. Then by definition $\Gamma\left(U \sim D, \mathscr{H}^{1}\right)=$ $H^{1}\left(A_{t}, R \otimes_{Z} Q\right)$; and by (3.5), $\Gamma\left(U \sim D, \mathscr{S}_{D}\right)=H^{0}\left(A / t A, R \otimes_{Z} Q\right)$. Further, the residue map:

$$
\text { res : } H^{1}\left(A_{t}, R \otimes_{z} Q\right) \longrightarrow H^{0}\left(\frac{A}{t A}, R \otimes_{z} Q\right)
$$

is precisely:

$$
\operatorname{res}_{D}: \Gamma\left(U \sim D, \mathscr{H}^{1}\right) \longrightarrow \Gamma\left(U \sim D, \mathscr{S}_{D}\right) .
$$

Suppose now that $(A, t)$ is an admissible pair. Let $U=\operatorname{spec} A$ and $D=V(t A)$. If $D$ is irreducible, then we may make the identifications of the previous paragraph, but $D$ need not be irreducible. We want to interpret the residue homomorphism:

$$
\text { res : } H^{1}\left(A_{t}, R \otimes_{z} Q\right) \longrightarrow H^{0}\left(\frac{A}{t A}, R \otimes_{z} Q\right)
$$

when $D$ is not necessarily irreducible. The basic idea is that the residue at $D$ of a cohomology class of $U \sim D$ is the direct sum of the residues on each irreducible component of $D$.

To make this precise, let $D_{1}, \cdots, D_{n}$ be the irreducible components of $D$. We shall assume that each $D_{i}$ is principal on $U$. Let $t_{i} A=I\left(D_{i}\right)$, and let $g_{i}: A / t A \rightarrow A / t_{i} A$ be the projection map. Since $A / t A$ is very smooth,

$$
\sum_{i=1}^{n} g_{i}: \frac{A}{t A} \longrightarrow \sum_{i=1}^{n} \oplus \frac{A}{t_{i} A}
$$

is bijective. Consequently each $A / t_{i} A$ is very smooth [8, Cor. to Th. 3.5]. That is, $U$ is an admissible neighborhood of each $D_{i}$. The induced map:

$$
\sum_{i=1}^{n} H^{0}\left(g_{i}\right): H^{0}\left(\frac{A}{t A}, R \otimes_{z} Q\right) \longrightarrow \sum_{i=1}^{n} \oplus H^{0}\left(\frac{A}{t_{i} A}, R \otimes_{Z} Q\right)
$$

is an isomorphism (5, Th. 5.7). Since 


$$
H^{0}\left(\frac{A}{t_{i} A}, R \otimes_{Z} Q\right)=\Gamma\left(U \sim D, \mathscr{S}_{D_{i}}\right)
$$

we have a canonical isomorphism:

$$
g_{*}: H^{0}\left(\frac{A}{t A}, R \otimes_{z} Q\right) \longrightarrow \sum_{i=0}^{n} \oplus \Gamma\left(U \sim D, \mathscr{S}_{D_{i}}\right) .
$$

This leads to a diagram which will relate the homomorphisms res and $\operatorname{res}_{D_{i}}$ :

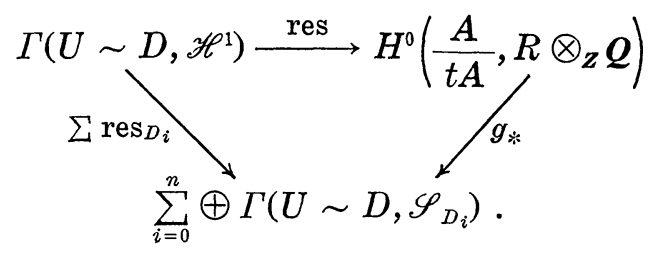

LEMMA 3. (1) is a commutative diagram.

Proof. It suffices for the lemma to show that the following diagram commutes :

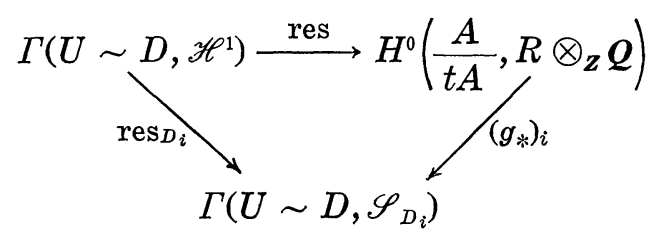

Let $B=A_{t_{1} \cdots \hat{t}_{i} \cdots t_{n}}$. Then $A_{t}=B_{t_{i}}$, and the inclusion map $f: A \rightarrow B$ is a map $f:(A, t) \rightarrow\left(B, t_{i}\right)$ of admissible pairs of ramification degree one [6, Def. 4.2]. Consequently we have a commutative square [6. Th. 4.3]:

$$
\begin{gathered}
H^{1}\left(A_{t}, R \otimes_{Z} Q\right) \stackrel{\text { res }_{t}}{\longrightarrow} H^{0}\left(\frac{A}{t A}, R \otimes_{Z} Q\right) \\
H^{1}(f) \mid \\
H^{1}\left(B_{t_{i}}, R \otimes_{Z} Q\right) \stackrel{H^{0}(f)}{\stackrel{\operatorname{res}_{t_{i}}}{\longrightarrow}} H^{0}\left(\frac{B}{t_{i} B}, R \otimes_{Z} Q\right) .
\end{gathered}
$$

The homomorphism $H^{1}(f)$ is the identity map. Further, $B / t_{i} B=A / t A$, as the primes $t_{i} A$ are comaximal. Thus $H^{0}(f)=\left(g_{*}\right)_{i}, \operatorname{res}_{t_{i}}=\operatorname{res}_{D_{i}}$, and the diagram (3) is just the same as (2), which proves the lemma.

The next lemma is an extension of (3.4). It demonstrates an important local property of ground field extension. 
LEMMA 4. Let $D$ be a prime divisor on $X$ and let $K$ be a finite extension of $k$. Suppose $U$ is an affine neighborhood of the generic point of $D$. Let $D_{1}, \cdots, D_{n}$ be the irreducible components of $K \times_{k} D$. There exists an admissible neighborhood $V \subset U$ of $D$ such that $V \times_{k} K$ is an admissible neighborhood (over $K$ ) of each $D_{i}$.

Proof. Choose $W \subset U$ to be an admissible neighborhood of $D$ (3.4). Let $A=\Gamma\left(W, \mathcal{O}_{X}\right)$ and $t A=I(D \cap W)$. Because $W$ is admissible, both $A$ and $A / t A$ are very smooth over $k$. Consequently, both $A \otimes_{k} K$ and $A / t A \otimes_{k} K$ are very smooth over $K$ (5, Th. 5.9).

Let $S=A \sim t A . \quad S$ is a multiplicative set since $D$ is irreducible. More importantly, $\left(A \otimes_{k} K\right)_{S}$ is a regular semilocal ring of height 1 whose maximal ideals correspond to the components $D_{i}$ of $D \times{ }_{k} K$. Thus there is an element $f \in S$ such that the ideal of each $D_{i}$ is principal in $\left(A \otimes_{k} K\right)_{f}$. Let $V=W_{f}$. $\quad V$ is an admissible neighborhood of $D$. We shall prove that $V \times{ }_{k} K$ is an admissible neighborhood of each $D_{i}$

Let $B=\Gamma\left(V \times{ }_{k} K, \mathcal{O}_{V \times{ }_{k} K}\right)=A_{f} \otimes_{k} K$, and set $I\left(V \times_{k} K \cap D_{i}\right)=t_{i} B$. No $t_{i}$ is a unit, and the ideals $t_{i} B$ are the minimal primes of $t B$. Both $B$ and $B / t B$ are very smooth over $K$, as they are localizations of very smooth rings. Since $B / t B$ is very smooth, each $B / t_{i} B$ is very smooth [8, Cor. to Th. 3.5]. Thus $V \times_{k} K$ satisfies the conditions required by the lemma.

Retain the conditions and notation of Lemma 4, and assume in addition that $U$ is very smooth affine and $D$ is principal on $U$. Let $S$ be a weak formalization of $K$. We shall use $U_{K}$ to denote $U \times{ }_{k} K, D_{K}$ to denote $D \times{ }_{k} K$, etc. Note that $U_{K}$ is considered as a $K$-variety, and that all $M-W$ cohomology sheaves on $V$ are formed with respect to $S$. We will prove that if every map

$$
\operatorname{res}_{D_{i}}: \Gamma\left(U_{K} \sim D_{i}, \mathscr{H}^{1}\right) \longrightarrow \Gamma\left(U_{K} \sim D_{i}, \mathscr{S}_{D_{i}}\right)
$$

is surjective, then

$$
\operatorname{res}_{D}: \Gamma\left(U \sim D, \mathscr{H}^{1}\right) \longrightarrow \Gamma\left(U \sim D, \mathscr{S}_{D}\right)
$$

is surjective. This result, stated as Corollary 6, is the key to proving the theorem announced at the beginning of this section.

In order to prove this result, we will first construct isomorphisms $\psi$ and $\rho$ which make the diagram below commute: 


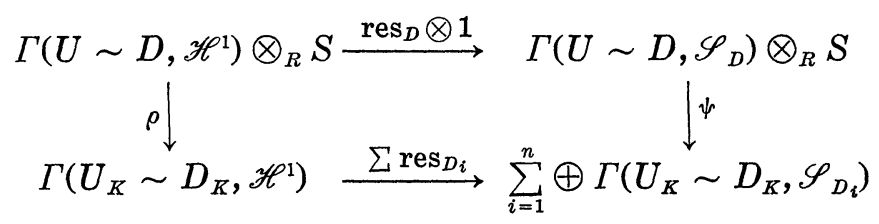

We will also prove that if $\operatorname{res}_{D} \otimes 1$ is onto, $\operatorname{res}_{D}$ is onto. The desired corollary will then follow easily.

Let $A=\Gamma\left(U, \mathcal{O}_{X}\right), \quad t A=I(D \cap U)$, and $B=A \otimes_{k} K=\Gamma\left(U_{K}, \mathcal{O}_{U_{K}}\right)$. Both $A$ and $B$ are very smooth, and the inclusion map $A_{t} \rightarrow B_{t}$ induces an isomorphism [8, Th. 5.9]:

$$
\rho: H^{1}\left(A_{t}, R \otimes_{Z} Q\right) \otimes_{R} S \longrightarrow H^{1}\left(B_{t}, S \otimes_{Z} Q\right) .
$$

This is the first of the two required maps.

To construct $\psi$, choose an admissible neighborhood $V \subset U$ of $D$ such that $V_{K} \subset U_{K}$ is an admissible neighborhood of each $D_{i}$ (Lemma 4). Note that the restriction maps:

$$
\begin{aligned}
& r: \Gamma\left(U \sim D, \mathscr{S}_{D}\right) \longrightarrow \Gamma\left(V \sim D, \mathscr{S}_{D}\right) \\
& r_{i}: \Gamma\left(U_{K} \sim D_{K}, \mathscr{S}_{D_{i}}\right) \longrightarrow \Gamma\left(V_{K} \sim D_{K}, \mathscr{S}_{D_{i}}\right)
\end{aligned}
$$

are bijective. Because these maps are bijective, we may construct $\psi$ over $V$ instead of over $U$.

Let $A^{\prime}=\Gamma\left(V, \mathcal{O}_{X}\right), B^{\prime}=A^{\prime} \otimes_{k} K=\Gamma\left(V_{K}, \mathcal{O}_{U_{K}}\right)$, and $t_{i} B^{\prime}=I\left(D_{i} \cap V_{K}\right)$. We still have $t A^{\prime}=I(V \cap D)$ and $t B^{\prime}=I\left(D_{K} \cap V_{K}\right) . A^{\prime} / t A^{\prime}, B^{\prime} / t B^{\prime}$, and $B^{\prime} / t_{i} B^{\prime}$ are all very smooth, and the natural map $f: A^{\prime} / t A^{\prime} \rightarrow B^{\prime} / t B^{\prime}$ induces an isomorphism [8, Th. 5.9]:

$$
H^{0}(f) \otimes 1: H^{0}\left(\frac{A^{\prime}}{t A^{\prime}}, R \otimes_{z} Q\right) \otimes_{R} S \longrightarrow H^{0}\left(\frac{B^{\prime}}{t B^{\prime}}, S \otimes_{z} Q\right)
$$

From (3.5), $H^{0}\left(A^{\prime} / t A^{\prime}, R \otimes_{Z} Q\right)=\Gamma\left(U \sim D, \mathscr{S}_{D}\right.$; and from Lemma 3 there is the isomorphism

$$
g_{*}: H^{0}\left(\frac{B^{\prime}}{t B^{\prime}}, S \otimes_{z} Q\right) \longrightarrow \sum_{i=1}^{n} \oplus \Gamma\left(V_{K} \sim D_{K}, \mathscr{S}_{D_{i}}\right)
$$

Let $\psi=g_{*} \circ\left(H^{0}(f) \otimes 1\right)$.

In order to show that (4) commutes, we consider first the square: 
(5)

$$
\begin{gathered}
H^{1}\left(A_{t}^{\prime}, R \otimes_{Z} Q\right) \otimes_{R} S \stackrel{\text { res } \otimes 1}{\longrightarrow} H^{0}\left(\frac{A^{\prime}}{t A^{\prime}}, R \otimes_{Z} Q\right) \otimes_{R} S \\
H^{1}(h) \downarrow \\
H^{1}\left(B_{t}^{\prime}, S \otimes_{Z} Q\right) \stackrel{\text { res }}{\longrightarrow} H^{0}\left(\frac{B^{\prime}(f) \otimes 1}{t B^{\prime}}, S \otimes_{Z} Q\right),
\end{gathered}
$$

where $H^{1}(h)$ is the cohomology homomorphism derived from the inclusion map $h: A^{\prime} \rightarrow B^{\prime}$. To see that (5) commutes, replace $H^{0}\left(A^{\prime} / t A^{\prime}, R \otimes_{Z} Q\right)$ $\otimes_{R} S$, by $H^{1}\left(D\left(A_{t}^{\prime}\right) / D\left(A^{\prime}\right)\right) \otimes_{Z} Q \otimes_{R} S$, and replace $H^{0}\left(B^{\prime} / t B^{\prime}, S \otimes_{Z} Q\right)$ by $H^{1}\left(D\left(A_{t}^{\prime}\right) / D\left(A^{\prime}\right)\right) \otimes_{R} S \otimes_{Z} Q$. These substitutions are permissible, as $S$ is a free $R$-module.

In terms of our sheaves and Lemma 3, (5) becomes

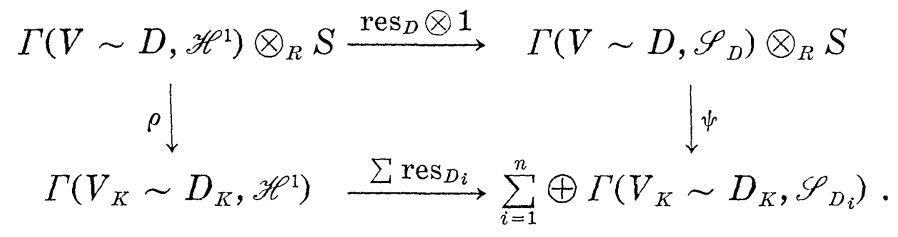

Diagram (6) is a special case of (4), where $V$ has been substituted for $U$. Taking account of the fact that the restriction maps $r$ and $r_{i}$ are bijective, the commutativity of (4) is immediate from (6).

Suppose now that $\operatorname{res}_{D} \otimes 1$ is surjective. Then $\operatorname{res}_{D}$ must itself be surjective, as $S$ is a free $R$-module.

We collect the above results into

LEMMA 5. Let $U$ be a very smooth affine neighborhood of the generic point of a prime divisor $D$ on $X$. Suppose $D \cap U$ is principal on $U$. Let $K$ be a finite extension of $k$, and let $U_{K}=U \times_{k} K . \quad U_{K}$ is considered as a K-variety, and all $M-W$ cohomology sheaves on $U_{K}$ are constructed with respect to a weak formalization $S$ of $K$ over $R$. Let $D_{1}, \cdots, D_{n}$ be the irreducible components of $D_{K}$ on $U_{K}$.

If the homomorphism:

$$
\sum \operatorname{res}_{D_{i}}: \Gamma\left(U_{K} \sim D_{K}, \mathscr{H}^{1}\right) \longrightarrow \sum_{i=1}^{n} \oplus \Gamma\left(U_{K} \sim D_{K}, \mathscr{S}_{D_{i}}\right)
$$

is surjective, then

$$
\operatorname{res}_{D}: \Gamma\left(U \sim D, \mathscr{H}^{1}\right) \longrightarrow \Gamma\left(U \sim D, \mathscr{S}_{D}\right)
$$

is surjective. 
COROLlaRY 6. Situation as in Lemma 5. If each homomorphism

$$
\operatorname{res}_{D_{i}}: \Gamma\left(U_{K} \sim D_{i}, \mathscr{H}^{1}\right) \longrightarrow \Gamma\left(U_{K} \sim D_{i}, \mathscr{S}_{D_{i}}\right)
$$

is surjective, then

$$
\operatorname{res}_{D}: \Gamma\left(U \sim D, \mathscr{H}^{1}\right) \longrightarrow \Gamma\left(U \sim D, \mathscr{S}_{D}\right)
$$

is surjective.

Proof. By Lemma 5, all we need prove is that

$$
\sum \operatorname{res}_{D_{i}}: \Gamma\left(U_{K} \sim D_{K}, \mathscr{H}^{1}\right) \longrightarrow \sum_{i=1}^{n} \oplus \Gamma\left(U_{K} \sim D_{K}, \mathscr{S}_{D_{i}}\right)
$$

is surjective. Let $\left(\tau_{i}\right) \in \sum_{i=1}^{n} \oplus \Gamma\left(U_{K} \sim D_{K}, \mathscr{S}_{D_{i}}\right)$. Choose $\sigma_{i} \in \Gamma\left(U_{K} \sim D_{i}, \mathscr{H}^{1}\right)$ such that $\operatorname{res}_{D_{i}}\left(\sigma_{i}\right)=\tau_{i}$. Note that since $D_{i} \not \subset D_{j}$ all $i \neq j, \Gamma\left(U_{K} \sim D_{i}, \mathscr{S}_{D_{j}}\right)$ $=0$. Consequently, $\operatorname{res}_{D_{j}}\left(\sigma_{i}\right)=0$, all $i \neq j$. Viewing each $\sigma_{i}$ as an element of $\Gamma\left(U_{K} \sim D_{K}, \mathscr{H}^{1}\right)$, let $\sigma=\sum_{i=1}^{n} \sigma_{i}$. Then $\sum_{i=1}^{n} \operatorname{res}_{D_{i}}(\sigma)=\left(\tau_{i}\right)$.

The next lemma is used to show that a divisor may be split up into absolutely irreducible components by a finite extension of the ground field.

LEMMA 7. Let $A$ be a finitely generated, integrally closed domain over a field $k$, and let $k^{\prime}$ be the algebraic closure of $k$ in $A$. Suppose $k^{\prime}$ is separable over $k$, and suppose $K$ is a finite separable extension containing a splitting field of $k^{\prime}$ over $k$. Let $\mathfrak{p}$ be a minimal prime of $A \otimes_{k} K$. Then $K$ is algebraically closed in $\left(A \otimes_{k} K\right) / \mathfrak{p}$.

Proof. $F=\left(A \otimes_{k} K\right) / \mathfrak{p}$ is a free join of $A$ and $X$ over $k$. Since $K$ contains a splitting field of $k^{\prime}, F$ is also a free join of $A$ and $K$ over $k^{\prime}$, provided we identify $k^{\prime}$ with an appropriate conjugate in $K$. Because $k^{\prime}$ is algebraically closed in the quotient field of $A$ and $K$ is separable over $k^{\prime}, F=A \otimes_{k^{\prime}} K$. Thus $K$ is algebraically closed in $F$. [cf. 10, Chap. III, Th.'s 37, 39, 40.]

The preliminaries are finished, and we come to the main result of this section.

THEOREM 8. Let $D$ be a prime divisor on $X$ and $p \in D$. There exists a neighborhood $U$ of $p$ such that the homomorphism:

$$
\operatorname{res}_{D}: \Gamma\left(U \sim D, \mathscr{H}^{1}\right) \longrightarrow \Gamma\left(U \sim D, \mathscr{S}_{D}\right)
$$

is surjective. 
Proof. Let $K$ be the splitting field of $K_{D}$ over $k$, and let $S$ be a weak formalization of $K$ over $R . \quad K_{D}$ and $K$ are separable over $k$, because $k$ is perfect. Since $\mathcal{O}_{X, p}$ is regular, there is a very smooth affine neighborhood $U \subset X$ of $p$ such that:

(1) $D \cap U$ is principal on $U$;

(2) $D \times{ }_{k} K$ has only principal components on $U \times{ }_{k} K$.

Set $V=U \times{ }_{k} K$, and let $D_{1}, \cdots, D_{n}$ be the irreducible components of $D \times{ }_{k} K . \quad V$ is considered as a $K$-variety, and all $M-W$ cohomology sheaves on $V$ are taken with respect to $S$.

By Corollary 6, it suffices to prove that

$$
\operatorname{res}_{D_{i}}: \Gamma\left(V \sim D_{i}, \mathscr{H}^{1}\right) \longrightarrow \Gamma\left(V \sim D_{i}, \mathscr{S}_{D_{i}}\right)
$$

is surjective for each $i$. We will show that $K_{D_{i}}=K$, which by Corollary 2 implies that $\operatorname{res}_{D_{i}}$ is surjective.

Let $U^{\prime} \subset U$ be an admissible neighborhood of $D$ such that $V^{\prime}=U^{\prime} \times{ }_{k} K$ is an admissible neighborhood of each $D_{i}$ (Lemma 4). Define $A=\Gamma\left(U^{\prime}, \mathcal{O}_{X}\right)$, $B=\Gamma\left(V^{\prime}, \mathcal{O}_{V}\right)=A \otimes_{k} K, t A=I\left(D \cap U^{\prime}\right)$, and $t_{i} B=I\left(D_{i} \cap V^{\prime}\right)$. Recall that $K_{D}$ is the algebraic closure of $k$ in $A / t A$, and $K_{D_{i}}$ is the algebraic closure of $K$ in $B / t_{i} B . \quad A / t A$ is integrally closed, so-by Lemma $7-K$ is algebraically closed in $B / t_{i} B$. That is, $K_{D_{i}}=K$.

\section{The sheaf $\mathscr{H}^{1}$}

The results of the foregoing sections permit us some theorems describing the cohomology of $\mathscr{H}^{1}$. As previously, $\left(X, \mathcal{O}_{X}\right)$ is a connected, smooth prescheme of finite type over a perfect field $k$, and $R$ is a complete, discrete valuation ring with residue class field $k$. As in 4 , we will use finite ground field extension to facilitate the following proofs.

LEMMA 1. Let $U \subset X$ be an affine complete transversal intersection, and let $D$ be a principal hypersurface on $U$. There exists a finite extension $K$ of $k$ and principal hypersurfaces $D_{1}, \cdots, D_{n}$ on $U \times_{k} K$ such that if $U_{0}=U \times{ }_{k} K$, and $U_{i}=U \times{ }_{k} K \sim \bigcup_{j=1}^{i} D_{j}$ :

(1) $\Gamma\left(U_{i}, \mathcal{O}_{U_{0}}\right)$ is a complete transversal intersection;

(2) $\Gamma\left(U_{i} \cap D_{i+1}, \mathcal{O}_{D_{i+1}}\right)$ is a complete transversal intersection;

(3) $D \times{ }_{k} K=D_{n}$.

If we set $V=U \sim D$ and $V_{0}=V \times_{k} K$, we may conclude from the lemma: there exists a decreasing sequence of open sets $U_{0} \supset U_{1} \supset \ldots$ 
$\supset U_{n} \subset V_{0}$ such that if $D_{i}=U_{i=1} \sim U_{i}$, then $D_{i}$ is a principal hypersurface on $U_{i-1}$, and $\left(\Gamma\left(U_{i-1}, \mathcal{O}_{U_{0}}\right), I\left(U_{i-1} \cap D_{i}\right)\right)$ is an admissible pair. In the case where $K=k$, this means that we may cut out of $U$ a very smooth hypersurface, then from what is left a second very smooth hypersurface, and so on, until what is left is a subset of $V$. Moreover, if $k$ is infinite, we may always take $K=k$.

Proof. It is not difficult to show that, if $A$ is a finitely generated smooth algebra over $k$ and if $f \in A$ is such that $A / f A$ is reduced, then there exists a finite extension $K$ of $k$ and non-zero-divisors $t_{0}=1, t_{1}, \cdots, t_{n-1}$, $t_{n}=t$ of $A \otimes_{k} K$ such that

$$
\frac{\left(A \otimes_{k} K\right)_{t_{0} \cdots t_{i-1}}}{\left(t_{i}\right)}
$$

is a smooth $k$-algebra, $1 \leq i \leq n$. Thus there exist principal hypersurface $D_{i}$ such that $D_{n}=D \times{ }_{k} K$ and $\Gamma\left(U_{i} \cap D_{i+1}, \mathcal{O}_{D_{i+1}}\right)$ is a regular ring. Since $\Gamma\left(U_{i}, \mathcal{O}_{U_{0}}\right)$ is a localization of $\Gamma\left(U, \mathcal{O}_{X}\right) \otimes_{k} K, \Gamma\left(U_{i}, \mathcal{O}_{U_{0}}\right)$ is a complete transversal intersection. Thus $\Gamma\left(U_{i} \cap D_{i+1}, \mathcal{O}_{D_{i+1}}\right)$ is a regular ring which is a complete transversal intersection modulo a principal ideal. Moreover, $\operatorname{dim} \Gamma\left(U_{i} \cap D_{i+1}, \mathcal{O}_{D_{i+1}}\right)=\operatorname{dim} \Gamma\left(U_{i}, \mathcal{O}_{U_{0}}\right)-1$. Consequently, since $k$ is perfect, $\Gamma\left(U_{i} \cap D_{i+1}, \mathcal{O}_{D_{i+1}}\right)$ is a complete transversal intersection.

The next proposition shows that a cohomology class-that is, a local section of $\mathscr{H}^{1}$-is determined by its value on any non-empty open subset of its domain of definition.

Proposition 2. Let $U \subset X$ be a non-empty open set. The restriction morphism $r: \Gamma\left(X, \mathscr{H}^{1}\right) \rightarrow\left(U, \mathscr{H}^{1}\right)$ is injective.

Proof. Since $\mathscr{H}^{1}$ is a sheaf (1.2), it suffices to prove the proposition for $X$ an affine complete transversal intersections and $U$ a principal open subset of $X$. Let $A=\Gamma\left(X, \mathcal{O}_{X}\right)$ and $U=X_{t}$ for some $t \in A$. If $(A, t)$ is an admissible pair, then by the Gysin sequence the proposition is true.

If $(A, t)$ is not an admissible pair, we must resort to the construction given in Lemma 1 . Let $K$ be an appropriate extension of $k$. Set $X_{0}=X \times{ }_{k} K$ and $U_{0}=U \times{ }_{k} K$. Recall that, when considering a finite field extension, all $M-W$ cohomology sheaves on $X_{0}$ are constructed with respect to some fixed weak formalization $S$ of $K$. By Lemma 1, there exist open sets $X_{0} \supset \cdots \supset X_{n} \subset U_{0}$ such that the restriction maps $\Gamma\left(X_{i}, \mathscr{H}^{1}\right)$ $\rightarrow \Gamma\left(X_{i+1}, \mathscr{H}^{1}\right)$ are injective. Thus the restriction map: 


$$
\Gamma\left(X_{0}, \mathscr{H}^{1}\right) \longrightarrow \Gamma\left(U_{0}, \mathscr{H}^{1}\right)
$$

is injective. This last map can be rewritten as:

$$
r \otimes 1: \Gamma\left(X, \mathscr{H}^{1}\right) \otimes_{R} S \longrightarrow \Gamma\left(U, \mathscr{H}^{1}\right) \otimes_{R} S .
$$

Since $S$ is a free $R$-module, $r$ must then be injective.

As a converse to the previous proposition we next present a theorem which describes the maximal extension of a section of $\mathscr{H}^{1}$. A lemma is required.

LEMMA 3. Let $U \subset X$ be an affine complete transversal intersection, and let $V \subset U$ be open affine. Let $K$ be a finite extension of $k$, and let $S$ be a weak formalization of $K$ over $R$. Let $U_{0}=U \times_{k} K, V_{0}=V \times_{k} K$, and $\pi: U_{0} \rightarrow U$ be the natural projection. Suppose $\sigma \in \Gamma\left(V, \mathscr{H}^{1}\right)$ is such that for any prime divisor $D$ on $U, \operatorname{res}_{D} \sigma=0$. Then for any prime divisor $D^{\prime}$ on $U_{0}, \operatorname{res}_{D^{\prime}} \pi_{*}(\sigma)=0$.

Proof. Let $D^{\prime}$ be a prime divisor on $U_{0}, D=\pi\left(D^{\prime}\right)$, and $D^{\prime \prime}=\pi^{-1}(D)$. $D$ is a prime divisor on $U$, and $D^{\prime}$ is a component of $D^{\prime \prime}$. Denote the components of $D^{\prime \prime}$ by $D^{\prime}=D_{0}^{\prime \prime}, \ldots, D_{r}^{\prime \prime}$. According to the discussion preceeding (4.5), $\Gamma\left(V_{0}, \mathscr{H}^{1}\right)=\Gamma\left(V, \mathscr{H}^{1}\right) \otimes_{R} S$ and $\sum \operatorname{res}_{D_{j}^{\prime \prime}}=\operatorname{res}_{D} \otimes 1$. Under this identification, $\pi_{*}(\sigma)=\sigma \otimes 1$. Since by hypothesis, $\operatorname{res}_{D} \otimes 1(\sigma \otimes 1)$ $=0, \operatorname{res}_{D}, \pi_{*}(\sigma)=0$.

Theorem 4. Let $U \subset X$ be open, and let $\sigma \in \Gamma\left(U, \mathscr{H}^{1}\right)$. Then $\sigma$ extends to an element of $\Gamma\left(X, \mathscr{H}^{1}\right)$ if and only if, for every prime divisor $D \subset X, \operatorname{res}_{D} \sigma=0$.

Proof. Suppose $\sigma$ extends to $\Gamma\left(X, \mathscr{H}^{1}\right)$. Then $\operatorname{res}_{D} \sigma \in \Gamma\left(X, \mathscr{S}_{D}\right)$, and $\Gamma\left(X, \mathscr{S}_{D}\right)=0$.

To prove the opposite implication, assume first that $X$ is an affine complete transversal intersection and that $D=X \sim U$ is a principal hypersurface on $X$ with irreducible components $D_{i}$. Let $A=\Gamma\left(X, \mathcal{O}_{X}\right)$. If $t A=I(D)$ and $(A, t)$ is an admissible pair, then (4.3) and the exact Gysin sequence;

$$
0 \longrightarrow \Gamma\left(X, \mathscr{H}^{1}\right) \longrightarrow \Gamma\left(U, \mathscr{H}^{1}\right) \stackrel{\sum \operatorname{res}_{D_{i}}}{\longrightarrow} \sum_{i} \oplus\left(U, \mathscr{S}_{D_{i}}\right)
$$

shows that $\sigma$ extends to $\Gamma\left(X, \mathscr{H}^{1}\right)$. 
If $(A, t)$ is not an admissible pair, we shall construct an appropriate field extension $K$ of $k$ as in the proof of Proposition 2. Then we will show that $\sigma \otimes 1$ extends from $U \times{ }_{k} K$ to $X \times{ }_{k} K$, and that this extension is the image of a cohomology class on $X$.

Choose $K$ to satisfy Lemma 1 , and define $X_{0}=X \times{ }_{k} K$. Let $\pi: X_{0} \rightarrow X$ be the canonical projection, and fix a weak formalization $S$ of $K$ over $R$. As usual $X_{0}$ is considered as a $K$-variety, and all $M-W$ cohomology sheaves on $X_{0}$ are constructed with respect to $S$. Let $U_{0}=U \times_{k} K$, and construct on $X_{0}$ principal hypersurfaces $D_{1}, \cdots, D_{n}$ such that if $X_{i}=X_{0} \sim \bigcup_{j=1}^{i} D_{j}$, then $\left(\Gamma\left(X_{i}, \mathcal{O}_{X_{0}}\right), I\left(D_{i+1} \cap X_{i}\right)\right)$ is an admissible pair, and $U_{0} \supset X_{n}$.

By Lemma $3, \operatorname{res}_{D^{\prime}} \pi_{*}(\sigma)=0$ for any prime divisor $D^{\prime}$ on $X_{0}$. Consequently we may use (4.3) and the exact Gysin sequence to prove that if $\pi_{*}(\sigma) \in \Gamma\left(X_{i}, \mathscr{H}^{1}\right)$, then $\pi_{*}(\sigma)$ extends to an element of $\Gamma\left(X_{i-1}, \mathscr{H}^{1}\right)$. Thus we have shown that $\pi_{*}(\sigma)$ is defined on $X_{0}$. Since $\Gamma\left(X_{0}, \mathscr{H}^{1}\right)=\Gamma\left(X, \mathscr{H}^{1}\right)$ $\otimes_{R} S$, and $\Gamma\left(X, \mathscr{H}^{1}\right) \subset \Gamma\left(U, \mathscr{H}^{1}\right), \sigma \in \Gamma\left(X, \mathscr{H}^{1}\right)[10$, 1.3.5.10]. Thus we have proven the theorem in the case that $X$ is a complete transversal intersection and $X \sim U$ is a principal hypersurface on $X$.

In general, replace $U$ by an open subset of $U$ such that $X \sim U=D$ is a hypersurface. Then $X$ can be covered by affine complete transversal intersections $W_{i}$, where either $W_{i} \subset U$ or $D \cap W_{i}$ is principal on $W_{i}$. In either case, $\sigma$ extends to an element $\sigma_{i} \in \Gamma\left(W_{i}, \mathscr{H}^{1}\right)$. By Proposition 2, the $\sigma_{i}$ patch together to extend $\sigma$ to $\Gamma\left(X, \mathscr{H}^{1}\right)$.

Recall (3.6), where we defined sheaves $\mathscr{H}_{D}^{0}, \tilde{K}_{D}$, and $\mathscr{S}_{D}$. Let $\mathscr{D}$ be the collection of prime divisors on $X$, and define sheaves:

$$
\begin{aligned}
\mathscr{S} & =\sum_{D \in \mathscr{D}} \oplus \mathscr{S}_{D} \\
\tilde{K} & =\sum_{D \in \mathscr{D}} \oplus \tilde{K}_{D} \\
\mathscr{T} & =\sum_{D \in \mathscr{D}} \oplus \mathscr{H}_{D}^{0} .
\end{aligned}
$$

We have an exact sequence:

$$
0 \longrightarrow \mathscr{S} \longrightarrow \tilde{K} \longrightarrow \mathscr{T} \longrightarrow 0 \text {. }
$$

LEMMA 5. For every open set $U \subset X$ and $i>0, H^{i}(U, \mathscr{S})=0$.

Proof. By [2, II.4.12.1], $H^{i}(U, \tilde{K})=\sum_{D \in \mathscr{D}} \oplus H^{i}\left(U, \tilde{K}_{D}\right)$, and $H^{i}(U, \mathscr{T})$ $=\sum_{D \in \mathscr{D}} \oplus H^{i}\left(U, \mathscr{H}_{D}^{0}\right)$ for $i \geq 0$. Thus $H^{i}(U, \tilde{K})=0$ and $H^{i}(U, \mathscr{T})=0$ 
for $i>0$. Also, $\Gamma(U, \tilde{K}) \rightarrow \Gamma(U, \mathscr{T})$ is surjective, because $\Gamma\left(U, \tilde{K}_{D}\right) \rightarrow$ $\Gamma\left(U, \mathscr{H}_{D}^{0}\right)$ is surjective for each $D \in \mathscr{D}$. The lemma follows.

By (3.7), the map:

$$
\sum_{D \in \mathscr{D}} \operatorname{res}_{D}: \mathscr{H}^{1} \longrightarrow \mathscr{S}
$$

is a morphism of sheaves. Let $\mathscr{C}=\operatorname{ker}\left(\sum \operatorname{res}_{D}\right)$ and $\mathscr{C}^{\prime}=\operatorname{coker}\left(\sum \operatorname{res}_{D}\right)$. We shall prove that $\mathscr{C}$ and $\mathscr{C}^{\prime}$ are constant sheaves.

LEMMA 6. $\mathscr{C}$ is a constant sheaf with stalk $\Gamma\left(X, \mathscr{H}^{1}\right)$.

Proof. Let $U \subset X$ be open. By Proposition 2 and Theorem 4, $\Gamma\left(X, \mathscr{H}^{1}\right)$ is precisely the kernel of the homomorphism $\sum \operatorname{res}_{D}: \Gamma\left(U, \mathscr{H}^{1}\right)$ $\rightarrow \Gamma(U, \mathscr{S})$.

Next we shall construct a constant sheaf which will turn out to be canonically isomorphic to $\mathscr{C}^{\prime}$.

Define a module:

$$
H=\frac{\underset{U \text { open }}{\stackrel{\lim }{\longrightarrow} \Gamma\left(U, \mathscr{H}^{1}\right)}}{\Gamma\left(X, \mathscr{H}^{1}\right)},
$$

and let $\tilde{H}$ be the constant sheaf with stalk $H$. The composite map for each open set $U$ :

$$
\Gamma\left(U, \mathscr{H}^{1}\right) \stackrel{\sum \operatorname{res}_{D}}{\longrightarrow} \Gamma(U, \mathscr{S}) \longrightarrow \Gamma(U, \tilde{K})
$$

has kernel equal to $\Gamma\left(X, \mathscr{H}^{1}\right)$. Consequently, this map induces a monomorphism $\psi: \tilde{H} \rightarrow \tilde{K}$.

Let $\mathscr{C}^{\prime \prime}=\operatorname{coker} \psi$. The canonical injection $\mathscr{S} \rightarrow \tilde{K}$ induces a morphism $\rho: \mathscr{C}^{\prime} \rightarrow \mathscr{C}^{\prime \prime}$, and we have a commutative diagram of sheaves:

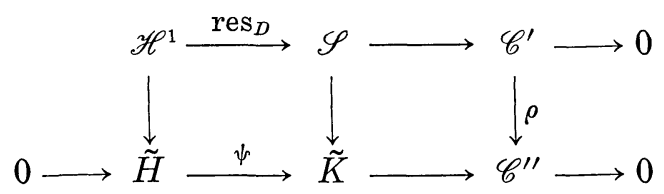

LEMMA 7. $\rho$ is bijective, so $\mathscr{C}^{\prime}=\mathscr{C}^{\prime \prime}$ is constant.

Proof. First we will prove that $\rho$ is injective. Suppose $U \subset X$ is open. Since $\Gamma(U, \mathscr{S}) \rightarrow \Gamma(U, \tilde{K})$ is injective, it suffices to show that 
$x \in \Gamma(U, \tilde{H}) \cap \Gamma(U, \mathscr{S})$ implies that $x$ pulls back to an element $y$ of $\Gamma\left(U, \mathscr{H}^{1}\right)$.

Because $x \in \Gamma(U, \tilde{H})$, there is an open set $V \subset U$ such that $x$ pulls back to an element $y \in \Gamma\left(V, \mathscr{H}^{1}\right)$. On the other hand, $x \in \Gamma(U, \mathscr{S})$ implies that for any prime divisor $D$ such that $D \cap U \neq \emptyset, \operatorname{res}_{D} y=0$. By Theorem $4, y$ extends to an element of $\Gamma\left(U, \mathscr{H}^{1}\right)$.

In order to prove that $\rho$ is surjective, we will show that for any point $p \in X, \mathscr{S}_{p}+\tilde{H}_{p}=\tilde{K}_{p}$. If $S_{D}$ is the module defined in (3.6), then:

$$
\begin{aligned}
\mathscr{S}_{p} & =\sum_{\substack{D \in \mathscr{D} \\
\boldsymbol{p} \in D}} \oplus S_{D} \otimes_{\boldsymbol{Z}} \boldsymbol{Q} \\
\tilde{K}_{p} & =\sum_{D \in \mathscr{D}} \oplus S_{D} \otimes_{\boldsymbol{Z}} \boldsymbol{Q} .
\end{aligned}
$$

Thus, to prove the lemma, it suffices to show that one can construct a cohomology class having prescribed residues on the prime divisors $D$ such that $p \in D$, provided that almost all of the prescribed residues are zero. The existence of this cohomology class will follow easily from the results of $\S 4$.

Suppose we prescribe residues $\tau_{i} \in S_{D_{i}} \otimes_{Z} \boldsymbol{Q}$ for some finite collection of $D_{i} \in \mathscr{D}$ passing through $p$. Using (4.8), select a neighborhood $U_{i}$ of $p$ and a cohomology class $\sigma_{i} \in \Gamma\left(U_{i} \sim D_{i}, \mathscr{H}^{1}\right)$ such that $\operatorname{res}_{D_{i}} \sigma_{i}=\tau_{i}$. If $D \neq D_{i}$ is another prime divisor passing through $p$, then $\operatorname{res}_{D} \sigma_{i}=0$. The cohomology class $\sigma=\sum_{i} \sigma_{i}$, defined on $\bigcap_{i} U_{i} \sim \cup_{i} D_{i}$, has the prescribed residues $\tau_{i}$ on $D_{i}$ and zero residue on every other prime divisor passing through $p$.

We conclude this section with a theorem which gives information about the cohomology of the sheaf $\mathscr{H}^{1}$. One short construction facilitates the statement of the theorem.

Let $\mathscr{L}$ be the sheaf $\operatorname{im}\left(\sum \operatorname{res}_{D}\right)$. We have two exact sequences of sheaves :

(1) $0 \longrightarrow \mathscr{C} \longrightarrow \mathscr{H}^{1} \longrightarrow \mathscr{L} \longrightarrow 0$

(2) $0 \longrightarrow \mathscr{L} \longrightarrow \mathscr{S} \longrightarrow \mathscr{C}^{\prime} \longrightarrow 0$

$\Gamma(X, \mathscr{S})=\Gamma(X, \mathscr{L})=0$, and by Lemma $5 H^{1}(X, \mathscr{S})=0$. Thus we may identify $\Gamma\left(X, \mathscr{C}^{\prime}\right)$ with $H^{1}(X, \mathscr{L})$. On the other hand, since $\mathscr{C}$ is constant, $H^{1}\left(X, \mathscr{H}^{1}\right) \cong H^{1}(X, \mathscr{L})$. If $U$ is open in $X$, let $\psi: \Gamma(U, \mathscr{S}) \rightarrow H^{1}\left(X, \mathscr{H}^{1}\right)$ be the composite map $\Gamma(U, \mathscr{S}) \rightarrow \Gamma\left(U, \mathscr{C}^{\prime}\right) \cong \Gamma\left(X, \mathscr{C}^{\prime}\right) \cong H^{1}\left(X, \mathscr{H}^{1}\right)$. 
THEOREM 8. Let $U \subset X$ be open, and let

$$
\begin{aligned}
& r: \Gamma\left(X, \mathscr{H}^{1}\right) \longrightarrow \Gamma\left(U, \mathscr{H}^{1}\right) \\
& r^{\prime}: H^{1}\left(X, \mathscr{H}^{1}\right) \longrightarrow H^{1}\left(U, \mathscr{H}^{1}\right)
\end{aligned}
$$

be the restriction morphisms. The following is a long exact sequence:

$$
\begin{aligned}
0 \longrightarrow \Gamma\left(X, \mathscr{H}^{1}\right) & \stackrel{r}{\longrightarrow} \Gamma\left(U, \mathscr{H}^{1}\right) \stackrel{\sum \operatorname{res}_{D}}{\longrightarrow} \Gamma(U, \mathscr{S}) \\
& \stackrel{\psi}{\longrightarrow} H^{1}\left(X, \mathscr{H}^{1}\right) \stackrel{r^{\prime}}{\longrightarrow} H^{1}\left(U, \mathscr{H}^{1}\right) \longrightarrow 0 .
\end{aligned}
$$

Consequently, coker $r^{\prime}$ and ker $r^{\prime}$ are both finite $R \otimes_{z} Q$-modules. Moreover, $H^{i}\left(X, \mathscr{H}^{1}\right)=0$ for $i \geq 2$.

Proof. Sequences (1) and (2), together with Lemmas 6 and 7, imply immediately that:

$$
\begin{aligned}
0 \longrightarrow \Gamma(U, \mathscr{C}) & \longrightarrow \Gamma\left(U, \mathscr{H}^{1}\right) \longrightarrow \Gamma(U, \mathscr{S}) \\
& \longrightarrow \Gamma\left(U, \mathscr{C}^{\prime}\right) \longrightarrow H^{1}(U, \mathscr{L}) \longrightarrow 0
\end{aligned}
$$

is exact. But $\Gamma(U, \mathscr{C})=\Gamma\left(X, \mathscr{H}^{1}\right), \Gamma\left(U, \mathscr{C}^{\prime}\right)=H^{1}\left(X, \mathscr{H}^{1}\right)$, and $H^{1}(U, \mathscr{L})$ $=H^{1}\left(U, \mathscr{H}^{1}\right)$. Consequently the proposed sequence is exact.

Coker $r$ and $\operatorname{ker} r^{\prime}$ are finite because $\Gamma(U, \mathscr{S})$ is finite. By Lemma 5, $H^{i}(U, \mathscr{S})=0$ for $i \geq 2$. Thus, by sequence (1), $H^{i}\left(U, \mathscr{H}^{1}\right)=0$ for $i \geq 2$.

Remark. Monsky has proven [7] that for any simple $k$-scheme $X$, $\Gamma\left(X, \mathscr{H}^{1}\right)$ is a finite $R \otimes_{Z} Q$-module. His proof is quite long, howeverby an easy application of $[5,5.4]$-if $X$ is projective and liftable to a projective smooth $R$-scheme then both $\Gamma\left(X, \mathscr{H}^{1}\right)$ and $H^{1}\left(X, \mathscr{H}^{1}\right)$ are finite dimensional $R \otimes_{z} Q$-modules.

\section{Applications}

\section{Algebraic Curves}

Suppose that the $k$-scheme $\left(X, \mathcal{O}_{X}\right)$ is a connected, absolutely nonsingular projective curve. Then $\left(X, \mathcal{O}_{X}\right)$ may be lifted to a flat, nonsingular, projective $R$-scheme $\left(X^{\prime}, \mathcal{O}_{X}^{\prime}\right)$ [9, pp. 35]. $\mathcal{O}_{X}^{\prime}$ possesses two important properties : its weak completion $\left(X^{\dagger}, \mathcal{O}_{X}^{\dagger}\right)$ is a weak formalization of $\left(X, \mathcal{O}_{X}\right)$; and $X^{\prime} \otimes_{z} Q=X^{*}$ is a nonsingular projective curve over the field $K=$ $R \otimes_{Z} Q$. Furthermore, if we assume that $k$ is algebraically closed in $\mathcal{O}_{X}$, then $K$ is algebraically closed in $\mathcal{O}_{X}^{*}$; also, the genus $g$ of $X$ equals the genus of $X^{*}[3]$. 
Let $\Omega_{X^{\prime} / R}^{1}$ be the sheaf of 1 -forms on $X^{\prime}$. Then the sheaf of 1 -forms $\Omega_{X^{\dagger} / R}^{1}$ on $X^{\dagger}$ may be identified with the weak completion of $\Omega_{X^{\prime} / R}^{1}$ (1.1). Also, $\Omega_{X^{\prime} / R}^{1} \otimes_{Z} Q=\Omega_{X^{*} / K}^{1}$ is the sheaf of 1-forms on $X^{*}$. Let $B^{*}$ (resp. $B^{\dagger}$ ) be the sheaf of exact 1-forms on $X^{*}$ (resp. $X^{\dagger}$ ). We will use the canonical identification $H^{i}\left(X^{*}, B^{*}\right)=H^{i}\left(X^{*}, \mathcal{O}_{X}^{*}\right), i \geq 1$, arising from the exact sequence of sheaves:

$$
0 \longrightarrow K \longrightarrow \mathcal{O}_{X}^{*} \longrightarrow B^{*} \longrightarrow 0 \text {; }
$$

where $K$ is the constant sheaf with stalk $K$. Similarly, we will identify $H^{i}\left(X^{\dagger}, B^{\dagger}\right)=H^{i}\left(X^{\dagger}, \mathcal{O}_{X}^{\dagger}\right), i \geq 1$.

We need two exact sequences: the first involves the group of differentials of the second kind $H=\Gamma\left(X^{*}, \Omega_{X^{*} / K}^{1} / B^{*}\right)$ over $X^{*}$. It is namely,

$$
0 \longrightarrow \Gamma\left(X^{*}, \Omega_{X^{*} / K}^{1}\right) \longrightarrow H \longrightarrow H^{1}\left(X^{*}, \mathcal{O}_{X}^{*}\right) \longrightarrow 0 \text {. }
$$

This sequence is exact, because $\operatorname{dim}_{K} H=2 g$, and $\operatorname{dim}_{K} \Gamma\left(X^{*}, \Omega_{X^{*} / K}^{1}\right)=$ $\operatorname{dim}_{K} H^{1}\left(X^{*}, \mathcal{O}_{X}^{*}\right)=g[1$, pp. 127, 130].

The second sequence is a dagger version of the first; it is the initial segment of the long exact cohomology sequence relating, $B^{\dagger}$, and $\mathscr{H}^{1}$ :

$$
0 \longrightarrow \Gamma\left(X^{\dagger}, \Omega_{X^{\dagger} / R}^{1}\right) \otimes_{\boldsymbol{Z}} \boldsymbol{Q} \longrightarrow \Gamma\left(X^{\dagger}, \mathscr{H}^{1}\right) \longrightarrow H^{1}\left(X^{\dagger}, \mathcal{O}_{X}^{\dagger}\right) \otimes_{\boldsymbol{Z}} \boldsymbol{Q} .
$$

Theorem 1. $\operatorname{Dim}_{K} H^{0}\left(X, \mathscr{H}^{1}\right)=2 g . \quad$ Also, $\operatorname{dim}_{K} H^{1}\left(X, \mathscr{H}^{1}\right)=1$.

Proof. Note that we may identify the sheaf $\mathscr{H}^{1}$ over $\left(X, \mathcal{O}_{X}\right)$ with the sheaf $\mathscr{H}^{1}$ over $\left(X^{\dagger}, \mathcal{O}_{X}^{\dagger}\right)$. Let $i: X^{\dagger} \rightarrow X^{\prime}$ be the canonical morphism. If $H$ is the collection of differentials of the second kind over $X^{*}$, we have a natural homomorphism $\alpha: H \rightarrow \Gamma\left(X^{\dagger}, \mathscr{H}^{1}\right)$ induced by the maps $\Omega_{X^{*} / K}^{1}$

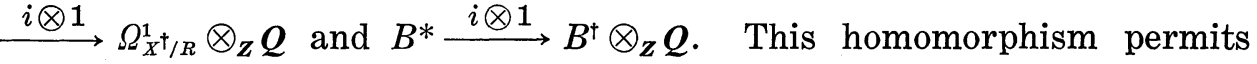
us to connect the two exact rows below into a commutative diagram:

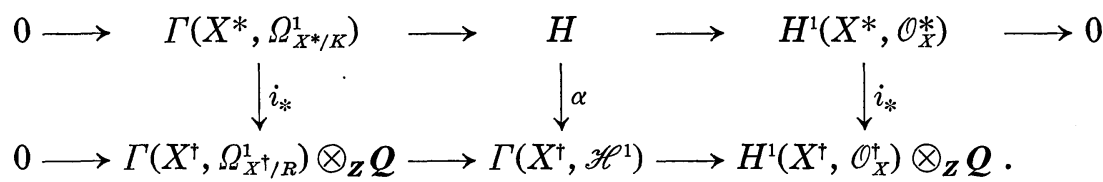

By the comparison theorem $[5,5.4]$, the maps $i_{*}$ are isomorphisms. Consequently, $\alpha$ is bijective, which implies $\operatorname{dim}_{K} \Gamma\left(X^{\dagger}, \mathscr{H}^{1}\right)=\operatorname{dim}_{K} H=2 g$.

Moreover, since $s$ is surjective, $s^{\dagger}$ is surjective. Thus the natural map $H^{1}\left(X^{\dagger}, \Omega_{X^{\dagger} / R}^{1}\right) \otimes_{Z} Q \rightarrow H^{1}\left(X, \mathscr{H}^{1}\right)$ is bijective (since $H^{i}\left(X^{\dagger}, \mathcal{O}_{X}^{\dagger}\right)=0$ for $i \geq 2$ by the comparison theorem). By the comparison theorem again, $\operatorname{dim}_{K} H^{1}\left(X^{\dagger}, \Omega_{X^{\dagger} / R}^{1}\right) \otimes_{Z} Q=\operatorname{dim}_{K} H^{1}\left(X^{*}, \Omega_{X^{*} / K}^{1}\right)=1$. 
COROLlaRY 2. Retain all of the above notation. Let $U \subset X$ be open, and let $X \sim U=\left\{P_{1}, \cdots, P_{r}\right\}$. The divisor $D=\sum_{i=1}^{r} P_{i}$ has degree $d g \cdot D=\sum_{i} \operatorname{dim}_{k}\left(\mathcal{O}_{P_{i}} / \mathfrak{m}_{P_{i}}\right)$. We have the following formula for the Euler characteristic of $\mathscr{H}^{1}$ on $U$ :

$$
\operatorname{dim}_{K} \Gamma\left(U, \mathscr{H}^{1}\right)-\operatorname{dim}_{K} H^{1}\left(U, \mathscr{H}^{1}\right)=2 g+d g \cdot D-1 .
$$

Proof. By the exact sequence of (5.8),

$$
\operatorname{dim}_{K} \Gamma\left(U, \mathscr{H}^{1}\right)=\operatorname{dim}_{K} H^{1}\left(U, \mathscr{H}^{1}\right)=2 g-1+\operatorname{dim}_{K} \Gamma(U, \mathscr{S}) .
$$

Since $\Gamma(U, \mathscr{S})=\sum_{i=1}^{r} \Gamma\left(U, \mathscr{S}_{P_{i}}\right)$, it suffices to prove that $\operatorname{dim}_{K} \Gamma\left(U, \mathscr{S}_{P_{i}}\right)$ $=\operatorname{dim}_{K} S_{P i} \otimes_{Z} Q=\operatorname{dim}_{k}\left(\mathcal{O}_{P_{i}} / \mathfrak{m}_{P i}\right)$. Since $S_{P_{i}}$ is the weak formalization of $\mathcal{O}_{P_{i}} / \mathfrak{m}_{P_{i}}$, the necessary equality follows from [8, Lemma 7.2].

Remark. If $A$ is an absolutely non-singular, finitely generated, onedimensional $k$-algebra, then $H^{1}$ (spec $A, \mathscr{H}^{1}$ ) $=0$. Consequently, the proceeding lemma gives us a technique for computing $\operatorname{dim}_{K} H^{1}\left(A ; R \otimes_{Z} Q\right)$. In particular, suppose $k$ is algebraically closed. Then $\operatorname{dim}_{K} H^{1}\left(A ; R \otimes_{Z} Q\right)$ is just $2 g+m-1$, where $m$ is the number of points needed to complete spec $A$ to a projective curve. This is the result suggested by topological consideration. A more computational proof of this result is given in [9, Th. 15.12].

\section{Further Numerical Results}

Let $\left(X^{\prime}, \mathcal{O}_{X}^{\prime}\right)$ be a smooth scheme over $R$, with weak completion $\left(X^{\dagger}, \mathcal{O}_{X}^{\dagger}\right)$ and reduction modulo $\mathfrak{m}\left(X, \mathcal{O}_{X}\right)$. If $\underline{\mathscr{H}}^{i}, i \geq 0$, denote the cohomology sheaves of the complex of $\mathcal{O}_{X}^{\dagger}$-modules $\Omega_{X^{\dagger} / R}^{\cdot} \otimes_{Z} Q$, we have the spectral sequence:

$$
E_{2}^{p q}=H^{p}\left(X^{\dagger}, \widetilde{\mathscr{H}}^{q}\right) \Rightarrow H^{n}\left(X^{\dagger}, \Omega_{X^{\dagger} / R}^{\cdot} \otimes_{Z} Q\right)
$$

Moreover, the topological space $X^{\dagger}$ may be identified with the topological space $X$, and via this identification there is a canonical isomorphism of sheaves $\underline{\mathscr{H}}^{1} \cong \widetilde{\mathscr{H}}^{1}$ and $\underline{\mathscr{H}}^{2} \cong \hat{\mathscr{H}}^{2}$ (cf. $\left.\S \S 1,2\right)$. Thus the spectral sequence may be written

$$
E_{2}^{p q}=H^{p}\left(X, \widetilde{\mathscr{H}}^{q}\right) \Rightarrow H^{n}\left(X^{\dagger}, \Omega_{X^{\dagger} / R} \otimes_{Z} Q\right)
$$

Proposition 4. (a) The canonical map $H^{1}\left(X^{\dagger}, \Omega_{X^{\dagger} / R} \otimes_{Z} Q\right) \rightarrow \Gamma\left(X, \mathscr{H}^{1}\right)$ arising from the spectral sequence is bijective.

(b) We have the following exact sequence, where the maps are all natural (that is, they commute with morphisms of $R$-schemes): 


$$
0 \longrightarrow H^{1}\left(X, \mathscr{H}^{1}\right) \longrightarrow H^{2}\left(X^{\dagger}, \Omega_{X^{\dagger} / R} \otimes_{\boldsymbol{Z}} Q\right) \longrightarrow \Gamma\left(X, \widetilde{\mathscr{H}}^{2}\right) \longrightarrow 0
$$

Proof. In our spectral sequence, $E_{2}^{i, j}=0$ if any of the following conditions are satisfied (5.8):

$$
\begin{aligned}
& j<0 \\
& j<0 \\
& j=0 \text { and } i \geq 1 \\
& j=1 \text { and } i \geq 2 .
\end{aligned}
$$

Thus (a) and (b) follow immediately.

Suppose $X^{\prime}$ is a projective smooth $R$-scheme. Let $X^{*}=X^{\prime} \times{ }_{Z} Q$. Then $\Omega_{X^{*} / R}^{\cdot} \otimes_{Z} Q=\Omega_{X^{\prime} / R}^{\cdot} \otimes_{Z} Q$, and $H^{1}\left(X^{*}, \Omega_{X^{*} / R}^{\cdot} \otimes_{Z} Q\right)=H^{1}\left(X^{\prime}, \Omega_{X^{\prime} / R}^{\cdot} \otimes_{Z} Q\right)$ $=H^{1}\left(X^{\dagger}, \Omega_{X^{\dagger} / R} \otimes_{Z} Q\right)=\Gamma\left(X, \mathscr{H}^{1}\right)$.

(The second equality follows from [5, 5.4], and the third from Proposition 4.) Similarly, we have the exact sequence:

$$
0 \longrightarrow H^{1}\left(X, \mathscr{H}^{1}\right) \longrightarrow H^{2}\left(X^{*}, \Omega_{X^{*} / R} \otimes_{Z} Q\right) \longrightarrow \Gamma\left(X, \widetilde{\mathscr{H}}^{2}\right) \longrightarrow 0
$$

COROLlary 5. If $g$ is the dimension of the Picard group of $X$, then $\operatorname{dim}_{K} \Gamma\left(X, \mathscr{H}^{1}\right)=2 g$.

COROLlaRY 6. If $V$ is a complex analytic manifold obtained from $X^{*}$ by imbedding a finitely generated field of definition for $X^{*}$ into $C$, then

$$
\operatorname{dim}_{K} \Gamma\left(X, \mathscr{H}^{2}\right)+\operatorname{dim}_{K} H^{1}\left(X, \mathscr{H}^{1}\right)=B_{2}(V),
$$

the second Betti number of $V$.

Proofs. Corollary 6 follows from the equality:

$$
\operatorname{dim}_{K} \Gamma\left(X, \mathscr{H}^{2}\right)+\operatorname{dim}_{K} H^{1}\left(X, \mathscr{H}^{1}\right)=\operatorname{dim}_{K} H^{2}\left(X^{*}, \Omega_{X^{* / R}} \otimes_{Z} Q\right)
$$

and $\left.\operatorname{dim}_{K} H^{2}\left(\Omega_{X^{*} / R}\right) \otimes_{Z} Q\right)=B_{2}(V)$, by [4].

To prove Corollary 5, we will show that $\operatorname{dim}_{K} H^{1}\left(\Omega_{X^{*} / R} \otimes_{Z} Q\right)=2 g$. If $g$ is the dimension of the Picard group of $X$, then $g$ is also the dimension of the Picard group of $X^{*}$ [3]. By (4) again, $\operatorname{dim}_{K} H^{1}\left(\Omega_{X^{*} / R}^{*} \otimes_{Z} Q\right)$ $=B_{1}(V)=2 g$.

Remark. Using the $l$-adic cohomology of Grothendieck, one can prove that $B_{2}(V)$ is independent of the choice of the lifting $X^{\prime}$ and the imbedding $X^{\prime} \rightarrow V$. Consequently, the conclusions of Corollaries 5 and 6 do not mention the lifting $X^{\prime}$, and one might conjecture that they remain true even when $\left(X, \mathcal{O}_{X}\right)$ has no global lifting. 
Birational Invariance of $\mathscr{H}^{1}$. We shall prove that the group of global cohomology classes of $\mathscr{H}^{1}$ is a birational invariant for complete, absolutely non-singular varieties over $k$.

Suppose that $K$ is a finitely generated extension of our perfect ground field $k$. Let $\mathscr{D}$ be the collection of prime divisors of $K / k$ (where a prime divisors is a discrete valuation ring in $K$ which has quotient field $K$ and contains $k$ ). If $\mathscr{U}$ is the collection of affine, non-singular models of $K / k$, we shall view $\mathscr{U}$ as an inductive set under the relation $U \leq V$ if and only if $\Gamma\left(U, \mathcal{O}_{U}\right) \subset \Gamma\left(V, \mathcal{O}_{V}\right)$ as subsets of $K$.

If $U \leq V$, then there is a natural injection:

$$
\psi_{U, V}: \Gamma\left(U, \mathscr{H}^{1}\right) \longrightarrow \Gamma\left(V, \mathscr{H}^{1}\right)
$$

as follows. The inclusion map $\Gamma(U) \rightarrow \Gamma(V)$ induces a continuous map $\mu: V \rightarrow U$. Let $\mathscr{A}$ be an open cover for $U$ and $\mathscr{B}$ be an open cover for $V$ subordinate to $\mu^{-1}(\mathscr{A})$. Since $k$ is perfect and $U$ and $V$ are nonsingular, $U$ and $V$ are smooth. Thus we may assume that $\mathscr{A}$ and $\mathscr{B}$ contain only very smooth affine open sets. If $U^{\prime} \in \mathscr{A}$ and $V^{\prime} \in \mathscr{B}$ are such that $\mu\left(V^{\prime}\right) \subset U^{\prime}$, then the associated homomorphism $\Gamma\left(U^{\prime}\right) \rightarrow \Gamma\left(V^{\prime}\right)$ induces a homomorphism

$$
\Gamma\left(U^{\prime}, \mathscr{H}^{1}\right) \longrightarrow \Gamma\left(V^{\prime}, \mathscr{H}^{1}\right)
$$

[8, Th. 5.6]. These homomorphisms patch together to give $\psi_{U, V}$. To show that $\psi_{U, V}$ is injective, choose each element of $\mathscr{B}$ to be a principal open subset of some element of $\mathscr{A}$. Recall (5.2), which shows that if $V^{\prime} \subset U^{\prime}$ is open, then $\Gamma\left(U^{\prime}, \mathscr{H}^{1}\right) \rightarrow \Gamma\left(V^{\prime}, \mathscr{H}^{1}\right)$ is injective. Consequently, $\psi_{U, V}$ is injective.

Since $\mathscr{U}$ is inductive, we may define:

$$
T=\underset{U \in \mathscr{\Psi}}{\lim } \Gamma\left(U, \mathscr{H}^{1}\right) \text {. }
$$

$T$ is analogous to the classical notion of one-dimensional cohomology classes of the third kind. Note that if $U^{\prime} \in \mathscr{U}$, then the natural map

$$
\underset{U \subset U^{\prime}}{\lim _{(1)}} \Gamma\left(U, \mathscr{H}^{1}\right) \longrightarrow T
$$

is bijective. The map is onto because the open sets $U \subset U^{\prime}$ are cofinal in $\mathscr{U}$; the map is injective because $\psi_{U, V}$ is injective.

If $D \in \mathscr{D}$, then $U \in \mathscr{U}$ is an admissible neighborhood of $D$ if and only if: 
(1) $D$ dominates a prime divisor $D^{\prime}$ on $U$;

(2) $U$ is an admissible neighborhood of $D^{\prime}$.

LEMMA 7. If $D \in \mathscr{D}$, then there is an admissible neighborhood of $D$.

Proof. If $U$ is an affine model of $K / k$ on which $D$ dominates a prime divisor, then $U$ contains an admissible neighborhood of $D$ (3.4). Thus it suffices to prove the following.

SUBLEMma. Let $K$ be a finitely generated extension of a perfect field $k$, and suppose $(S, \mathfrak{n})$ is a discrete valuation ring of $K / k$. There exists a regular, finitely generated k-algebra $A \subset S$ with quotient field $K$ such that $\mathfrak{n} \cap A$ has height one.

Proof. Let $n=t r \cdot d g \cdot{ }_{k} K$, and choose $x_{1}, \cdots, x_{n-1} \in S$ to be algebraically independent over $k$ in $S / \mathfrak{n}$. Define $C=k\left[x_{1}, \cdots, x_{n}\right]$, where $x_{n}$ is a generator for $\mathfrak{n}$ in $S$. Since $x_{n}$ is transcendental over $k\left(x_{1}, \cdots, x_{n-1}\right)$, $K$ is a finite extension of $k\left(x_{1}, \cdots, x_{n}\right)$. Let $B$ be the integral closure of $C$ in $K$. The quotient field of $B$ is $K$, and $t r \cdot d g \cdot{ }_{k}(B / \mathfrak{n} \cap B)=n-1$. Thus $\mathfrak{n} \cap B$ has height one. Since $B$ is integrally closed and $\mathfrak{n} \cap B$ has height one, there exists an element $f \in B \sim \mathfrak{n}$ such that $A=B_{f}$ is regular. A satisfies the sublemma.

Suppose $D \in \mathscr{D}$ and $(S, \mathfrak{n})$ is the ring of $D$. Let $k^{\prime}$ be the algebraic closure of $k$ in $S / \mathfrak{n}$, and define $K^{\prime}=H^{0}\left(k^{\prime} ; R \otimes_{Z} Q\right)$. Note that if $U$ is a model of $K / k$ such that $D$ dominates a prime divisor $D^{\prime}$ on $U$, then $S_{D^{\prime}}$ is a weak formalization over $R$ of $k^{\prime}$ (3.6). Moreover, $K^{\prime}=S_{D^{\prime}} \otimes_{Z} Q$.

Definition 8. Suppose $\omega \in T$. Then $\operatorname{res}_{D} \omega \in K^{\prime}$ is defined as follows : choose an admissible neighborhood $U$ of $D$ and an open affine subset $V \subset U$ such that $\omega \in \Gamma\left(V, \mathscr{H}^{1}\right)$. Let $D^{\prime}$ be the hypersurface on $U$ dominated by $D$. Set

$$
\operatorname{res}_{D} \omega=\operatorname{res}_{D^{\prime}} \omega \text {. }
$$

We must show that this definition does not depend on the choice of $U$. The definition is obviously independent of the choice of $V \subset U$. Note that the definition of $\operatorname{res}_{D}$ also remains invariant whenever we replace $U$ by a principal open subset of $U$ which is still an admissible neighborhood of $D$. Since any two admissible neighborhoods of $D$ contain principal open subsets which are admissible neighborhoods of $D$ and which 
may be identified with one another, $\operatorname{res}_{D} \omega$ is independent of the choice of $U$.

Proposition 9. Let $D \in \mathscr{D}$. Suppose $U$ is a model of $K / k$ such that $D$ dominates some point $P$ of $U$ (not necessarily a point of codimension one). If $\omega \in \Gamma\left(U, \mathscr{H}^{1}\right) \subset T$, then $\operatorname{res}_{D} \omega=0$.

Proof. If $P$ has codimension one, then the proposition follows immediately from (5.3). Otherwise choose $V$ to be an admissible neighborhood of $D$. Let $(S, \mathfrak{n})$ be the ring of $D . \quad \Gamma(U) \subset S$ and $\Gamma(V) \subset S$; further, $\mathfrak{n} \cap \Gamma(V)$ has height one. Since $V$ is nonsingular, $\Gamma(V)_{\mathfrak{n} \cap \Gamma(V)}=S$. Thus there exists $f \in \Gamma(V) \sim \mathfrak{n}$ such that $\Gamma(V)_{f} \supset \Gamma(U) . \quad V_{f}$ remains an admissible neighborhood of $D$, and $U \leq V_{f}$. Considering $\omega$ as an element of $\Gamma\left(V_{f}, \mathscr{H}^{1}\right)$, (5.3) proves that $\operatorname{res}_{D} \omega=0$.

THEOREM 10. If $X$ is a complete, non-singular model of $K / k$, then $\Gamma\left(X, \mathscr{H}^{1}\right)=\left\{\omega \in T ; \operatorname{res}_{D} \omega=0\right.$ for every $\left.D \in \mathscr{D}\right\}$. Consequently, $\Gamma\left(X, \mathscr{H}^{1}\right)$ is a birational invariant.

Proof. Let $T^{\prime}=\left\{\omega \in T ; \operatorname{res}_{D} \omega=0\right.$ for every $\left.D \in \mathscr{D}\right\}$. Proposition 9 shows that $\Gamma\left(X, \mathscr{H}^{1}\right) \subset T^{\prime}$; and (5.3) proves the opposite inclusion.

Remark. $\quad T^{\prime}$ is analogous to the differentials of the second kind on $X$. If $U$ and $V$ are both models of $K / k$ (complete or not), then $\Gamma\left(U, \mathscr{H}^{1}\right)$ and $\Gamma\left(V, \mathscr{H}^{1}\right)$ each contains a subgroup corresponding to $T^{\prime}$. We may extend the conjecture which follows Corollary 6, and suggest that perhaps

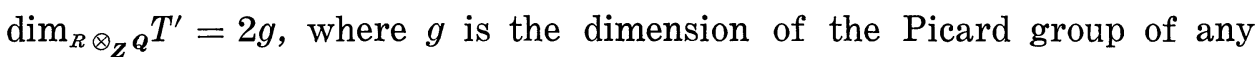
complete normal model for $K / k$. We must use normal model here, because $T^{\prime}$ can be defined even if $K / k$ has no complete nonsingular model.

\section{REFERENCES}

[1] Chevally, C., Introduction to the Theory of Algebraic Functions of One Variable. New York, Math. Surveys IV, 1951.

[2] Grothendieck, A., Eléments de Géométrie Algébrique.. Paris, IHES 4 and 11 (1960-61).

[ 3 ] Grothendieck, A., Techniques de Descente VI. Paris, Seminar Bourbaki, 1961-62.

[4] Grothendieck, A., On the De Rham Cohomology of Algebraic Varieties. Paris, IHES 29 (1966).

[ 5 ] Meredith, D., Weak Formal Schemes. Nagoya Math. J. 45 (1972), pp. 1-38.

[ 6 ] Monsky, P., Formal Cohomology II. Ann. of Math. 88 (1968), pp. 218-238.

[ 7 ] Monsky, P., Formal Cohomology IV. to appear.

[ 8 ] Monsky, P. and Washnitzer, G., Formal Cohomology I. Ann. of Math. 88 (1968), pp. 181-217. 
[9] Notes from a seminar given at Princeton University by Monsky and Washnitzer in 1964.

[10] Zariski, O. and Samuel, P., Commutative Algebra.. New York, Van Nostrand, 1958.

[11] Bourbaki, N., Algèbre Commutative (Chapitres I-IV). Paris, Actualités Scientifiques et Industrielles 1290 and 1293 (1961).

Mass. Inst. of Technology 